\title{
15. Nevanlinna's fundamental theorems and Ahlfors' theorem on the number of asymptotic values.
}

\author{
By Masatsugu TsusI.
}

(Received March 12, 1942.)

\section{PART I. NEVANLINNA'S FUNDAMENTAL THEOREMS.}

\section{First Fundamental Theorem.}

Let $K$ be the Riemann sphere of diameter 1, which touches the $w$-plane at $w=0$ and $[a, b]=\frac{|a-b|}{\sqrt{\left(1+|a|^{2}\right)\left(1+|b|^{2}\right)}}$ and $\Delta$ be a domain on the $z$-plane, which contains $\infty$ on its boundary and $w=w(z)=f(z)$ be onevalued and meromorphic in and at the boundary points $(\neq \infty)$ of $\lrcorner$, such that $\left[f(z), w_{0}\right]<\delta$ in $\Delta$ and $\left[f(z), w_{0}\right]=\delta$ at the boundary points $(\neq \infty)$ of $d$, so that the inverse function $z=\phi(w)$ of $w=f(z)$ is defined on the Riemann surface $F$, spread over $\left[w, w_{0}\right]<\delta$.

A $\delta$-neighbourhood $U$ of $w_{0}$ is defined as the connected part of $F$, which lies above $\left[w, w_{0}\right]<\delta$ and has $w_{0}$ as an inner point or as a boundary point. The boundary $A$ of $\Delta$ consists of two classes of curves. Namely the ones which extend to infinity and the others which are closed curves and form holes in $\Delta$. We add all such holes to $J$ and the resulting simply connected domain be denoted by $\bar{J}$. If $\bar{J}$ has a boundary curve which extends to infinity, let $\Lambda_{0}$ be the outermost such curve and the simply connected domain bounded by $\Lambda_{0}$ be denoted by $\overline{\bar{J}}$. Let $z_{0}$ be a fixed point. We denote the part of $\Delta$, which lies in $\left|z-z_{0}\right| \leqq r$ by $\Delta_{r}$ and the common part of $\left|z-z_{0}\right|=r$ and $4, \bar{A}, \overline{\bar{A}}$ by $\theta_{r}, \bar{\theta}_{r}, \overline{\bar{\theta}}_{r}$ and its total length by $r \theta(r), r \bar{\theta}(r), r \overline{\bar{\theta}}(r)$ respectively.

We put

$L(r)=L(r, w ; d)=$ the total length of the image of $\theta_{r}$ by $w=f(z)$ on $K$, $A(r)=A(r, w ; \Delta)=$ the total area of the image of $\Delta_{r}$ by $w=f(z)$ on $K$, $S(r)=S(r, w ; d)=A(r) / \pi \delta^{2}$, where $\pi \delta^{2}$ is the area of $\left[w, w_{0}\right]<\delta$ on $K$, $n(r, a)=n(r, a, w ; \Delta)=$ the number of zero points of $f(z)-a$ in $\Delta_{r}$, 


$$
\begin{aligned}
& N(r, a)=N(r, a, w ; \Delta)=\int_{r_{0}}^{r} \frac{n(r, a)}{r} d r \\
& m(r, a)=m(r, a, w ; \Delta)=\frac{1}{2 \pi} \int_{\theta_{r}} \log \frac{1}{\left[w\left(r e^{i \theta}\right), a\right]} d \theta \\
& T(r, a)=T(r, a, w ; \Delta)=N(r, a)+m(r, a)
\end{aligned}
$$

Then the following theorem( $\left.{ }^{1}\right)$ corresponds to Nevanlinna's first fundamental theorem.

Theorem I (FIRST FUNDAMENTAL THEOREM). For any point a in $\left[w, w_{0}\right]<\delta$,

$$
T(r, a)=T(r)+O\left(\int_{r_{0}}^{r} \frac{L(r)}{r} d r\right),
$$

where

$$
\begin{aligned}
T(r)= & T(r, w ; \Delta)=\int_{r_{0}}^{r} \frac{S(r)}{r} d r, \\
\int_{r_{0}}^{r} \frac{L(r)}{r} d r & =O(\sqrt{T(2 r) \log r}) \text { for all } r, \\
& =O(\sqrt{T(r)} \log T(r)),
\end{aligned}
$$

except certain intervals $1_{n}$, such that $\sum_{n} \int_{I_{n}} d \log \log r<\infty\left({ }^{2}\right)$.

We will call $T(r)$ the characteristic function of $f(z)$ in $\Delta$ and $\varlimsup_{r \rightarrow \infty} \frac{\log T(r)}{\log r}=\rho$ the order of $f(z)$ in $\Delta$. If $\rho<\infty$, then we have from (1), (2),

$$
T(r, a)=T(r)+O\left(r^{p^{\prime}}\right)\left(\rho^{\prime}<\rho\right)
$$

\section{Some lemmas.}

We will prove some lemmas.

Lemma. Let $w=w(z)=w\left(r e^{i \theta}\right)$ satisfy the condition in $\$ 1$ and $U(w)$ be a linear transformation, which makes $\left[w, w_{0}\right]<\delta$ invariant, then

$$
\int\left|\frac{d}{d r} \log \frac{[U(w), b]}{[w, b]}\right| d \omega(b) \leqq \text { const. } \frac{\left|w^{\prime}\right|}{1+|w|^{2}},
$$

(1) M. TsuJI : On the behaviour of an inverse function of a meromorphic function at its transcendental singular point, III. Proc. Imp. Acad. 18 (1942). Y. TumurA : Sur le problème de M. Kunugui. Proc. Imp. Acad. 18 (1942) and Sur le premiere théorème dans la théorie des fonctions méromorphes. Proc. Imp. Acad. 18 (1924).

(2) A. Dinghas: Eine Bemerkung zur Ahlforsschen Theorie der Überlagerungsflächen. Math. Z. 44 (1939). 


$$
\left|\frac{d}{d r} \log \frac{[U(w), U(b)]}{[w, b]}\right| \leqq \text { const. } \frac{\left|w^{\prime}\right|}{1+|w|^{2}}
$$

where $\left[b, w_{0}\right]<\delta$ and $d \omega(b)$ is the surface element on $K$ at $b$.

Proof. We may assume that $\left[w, w_{0}\right]<\delta$ is a circle $|w|<\rho$ and $U(w)=\frac{\rho(w-a)}{\rho^{2}-\bar{a} w}(|a|<\rho)$, since by the rotation of $K$, we can bring $\left[w, w_{0}\right]<\delta$ to $|w|<\rho$ and $\frac{\left|w^{\prime}\right|}{1+|w|^{2}}$ is invariant by the rotation.

Now

$$
\begin{aligned}
& \left|\frac{d}{d r} \log [w, b]\right| \leqq\left|\frac{d}{d r} \log \right| w-b||+\frac{1}{2}\left|\frac{d}{d r} \log \left(1+|w|^{2}\right)\right|, \\
& \left|\frac{d}{d r} \log \right| w-b|| \leqq\left|\frac{d}{d r} \log (w-b)\right|=\frac{\left|w^{\prime}\right|}{|w-b|},
\end{aligned}
$$

so that

$$
\int_{|b| \leq p}\left|\frac{d}{d r} \log \right| w-b|| d \omega(b) \leqq \text { const. }\left|w^{\prime}\right| \leqq \text { const. } \frac{\left|w^{\prime}\right|}{1+|w|^{2}} .
$$

If we put $w=u+i v$, then

$$
\frac{1}{2}\left|\frac{d}{d r} \log \left(1+|w|^{2}\right)\right|=\frac{\left|u u_{r}+v v_{r}\right|}{1+|w|^{2}} \leqq \frac{\sqrt{\left(u^{2}+v^{2}\right)\left(u_{r}^{2}+v_{r}^{2}\right)}}{1+|w|^{2}} \leqq \text { const. } \frac{\left|w^{\prime}\right|}{1+|w|^{2}} .
$$

Hence from (4), (5), (6),

Similarly

$$
\int_{|b| \leq p}\left|\frac{d}{d r} \log [w, b]\right| d \omega(b) \leqq \text { const. } \frac{\left|w^{\prime}\right|}{1+|w|^{2}} .
$$

$$
\int_{|b| \leq p}\left|\frac{d}{d r} \log [U(w), b]\right| d \omega(b) \leqq \text { const. }\left|\frac{d}{d z} U(w)\right| \leqq \text { const. } \frac{\left|w^{\prime}\right|}{1+|w|^{2}} .
$$

Hence we have from (7), (8),

$$
\int_{|b| \leq p}\left|\frac{d}{d r} \log \frac{[U(w), b]}{[w, b]}\right| d \omega(b) \leqq \text { const. } \frac{\left|w^{\prime}\right|}{1+|w|^{2}},
$$

which proves the first part. Next

$$
\begin{aligned}
\left|\frac{d}{d r} \log \frac{[U(w), U(b)]}{[w, b]}\right| & \leqq\left|\frac{d}{d r} \log \right| \frac{U(w)-U(b)}{w-b}|| \\
& +\frac{1}{12}\left|\frac{d}{d r} \log \left(1+|U(w)|^{2}\right)\right|+\frac{1}{2}\left|\frac{d}{d r} \log \left(1+|w|^{2}\right)\right| .
\end{aligned}
$$


Since

$$
\left|\frac{U(w)-U(b)}{w-b}\right|=\frac{\rho\left(\rho^{2}-|a|^{2}\right)}{\left|\rho^{2}-\bar{a} w\right| \cdot\left|\rho^{2}-\bar{a} b\right|},
$$

we have

$$
\left|\frac{d}{d r} \log \right| \frac{U(w)-U(b)}{w-b}|| \leqq \text { const. } \frac{\left|w^{\prime}\right|}{1+|w|^{2}}
$$

By (6),

$$
\frac{1}{2}\left|\frac{d}{d r} \log \left(1+|U(w)|^{2}\right)\right| \leqq \text { const. }\left|\frac{d}{d z} U(w)\right| \leqq \text { const. } \frac{\left|w^{\prime}\right|}{1+|w|^{2}} .
$$

From (9), (10), (11), (6), we have

$$
\left|\frac{d}{d r} \log \frac{[U(w), U(b)]}{[w, b]}\right| \leqq \text { const. } \frac{\left|w^{\prime}\right|}{1+|w|^{2}},
$$

which proves the second part.

Theorem II. Let $w=w(z)$ satisfy the condition in $\$ 1$ and $U(w)$ be a linear transformation, which makes $\left[w, w_{0}\right]<\delta$ invariant, then

$$
S(r, w ; \Delta)-S(r, U(w) ; \Delta)=O(L(r)) .
$$

Proof. Let $\Gamma_{r}$ be the whole boundary of $\Delta_{r}$ and $\Gamma_{r}=\theta_{r}+\gamma_{r}$ and $a, b$ be any two points in $\left[w, w_{0}\right]<\delta$, then

$$
\begin{aligned}
& \frac{1}{2 \pi} \int_{\theta_{r}} \frac{d}{d r} \log \frac{[w ; a]}{[w, b]} d \theta= \frac{1}{2 \pi} \int_{\theta_{r}} \frac{d}{d r} \log \frac{|w-a|}{|w-b|} d \theta \\
&= \frac{1}{2 \pi r} \int_{e_{r}} \operatorname{darg} \frac{w-a}{w-b}= \\
& \frac{1}{2 \pi r} \int_{\Gamma_{r}} \operatorname{darg} \frac{w-a}{w-b}-\frac{1}{2 \pi r} \int_{\gamma_{r}} \operatorname{darg} \frac{w-a}{w-b}=\frac{n(r, a, w ; \Delta)-n(r, b, w ; \Delta)}{r} \\
&-\frac{1}{2 \pi r} \int_{\gamma_{r}} \operatorname{darg} \frac{w-a}{w-b} .
\end{aligned}
$$

Now $\gamma_{r}$ consists of two classes of curves: $\gamma_{r}=\gamma_{r}^{(1)}+\gamma_{r}^{(2)}$, where $\gamma_{r}^{(1)}$ are closed curves and form holes of $\Delta_{r}$ and $\gamma_{r}^{(2)}$ are not closed curves and meet $\theta_{r}$. Evidently

$$
\int_{\gamma_{r}^{(1)}} \operatorname{darg} \frac{w-a}{w-b}=0
$$


Let $\Delta_{r}$ consists of a certain number of connected domains $\Delta_{r}^{(i)}(i=1,2, \ldots)$ and $\gamma_{r, i}^{(2)}+\theta_{r, i}$ be the part of $\gamma_{r}^{(2)}+\theta_{r}$, which belongs to the boundry of $\Delta_{r}^{(i)}$ and let its image on $K$ be of the form :

$$
\left(\alpha_{1}, \beta_{1}\right)+\Theta_{1}+\left(\alpha_{2}, \beta_{2}\right)+\Theta_{2}+\cdots+\left(\alpha_{n}, \beta_{n}\right)+\Theta_{n},
$$

where $\sum_{\nu=1}^{n}\left(\alpha_{\nu}, \beta_{\nu}\right)$ is the image of $\gamma_{r, i}^{(2)}$ and consists of circular arcs on $\left[w, w_{0}\right] \stackrel{\nu=1}{=} \delta$ and $\sum_{\nu=1}^{n} \Theta_{\nu}$ is the image of $\theta_{r, i}$ where $\Theta_{\nu}$ connects $\beta_{\nu}$ and $\alpha_{\nu+1}$ in $\left[w, w_{0}\right]<\delta,\left(\alpha_{n+1}=\alpha_{1}\right)$. We replace $\Theta_{\nu}$ by a circular are $\left(\beta_{\nu}, \alpha_{\nu+1}\right)$ on $\left[w, w_{0}\right]=\delta$ and put

$$
\begin{aligned}
& C=\left(\alpha_{1}, \beta_{1}\right)+\left(\alpha_{2}, \beta_{2}\right)+\cdots \cdots+\left(\alpha_{n}, \beta_{n}\right), \\
& C^{\prime}=\left(\beta_{1}, \alpha_{2}\right)+\left(\beta_{2}, \alpha_{3}\right)+\cdots+\left(\beta_{n}, \alpha_{1}\right), \\
& C^{\prime \prime}=C+C^{\prime},
\end{aligned}
$$

then $C^{\prime \prime}$ is an integral multiple of the whole circumsference of $\left[w, w_{0}\right]=\delta$, so that $\int_{C^{\prime \prime}} \operatorname{darg} \frac{w-a}{w-b}=0$, hence

$$
\begin{aligned}
\int_{\gamma_{r, i}^{(2)}} \operatorname{darg} \frac{w-a}{w-b}=\int_{C} \operatorname{darg} \frac{w-a}{w-b} & =\int_{C^{\prime \prime}} \operatorname{darg} \frac{w-a}{w-b}-\int_{C^{\prime}} \operatorname{darg} \frac{w-a}{w-b} \\
& =-\int_{C^{\prime}} \operatorname{darg} \frac{w-a}{w-b} .
\end{aligned}
$$

If $a, b$ lie in $\left[w, w_{0}\right] \leqq \delta_{1}<\delta$, then

$$
\left|\int_{C^{\prime}} \operatorname{darg} \frac{w-a}{w-b}\right| \leqq K\left(\delta_{1}\right) \times \text { sum of lengths of }\left(\Theta_{1}+\cdot+\Theta_{n}\right),
$$

where $\dot{K}\left(\delta_{1}\right)$ depends on $\delta_{1}$ only. Hence summing up for $i=1,2, \ldots$,

$$
\left|\int_{\gamma_{r}^{(2)}} \operatorname{darg} \frac{w-a}{w-b}\right| \leqq K\left(\delta_{1}\right) \cdot L(r),
$$

so that from (13), (15),

$$
\left|\frac{1}{2 \pi r} \int_{\gamma_{r}} \operatorname{darg} \frac{w-a}{w-b}\right| \leqq K\left(\delta_{1}\right) \cdot \frac{L(r)}{r} .
$$

Hence from (12), (16),

$$
\begin{aligned}
\frac{1}{2 \pi} \int_{\theta_{r}} \frac{d}{d r} \log \frac{[w, a]}{[w, b]} d \theta & \\
= & \frac{n(r, a, w ; \Delta)-n(r, b, w ; \Delta)}{r}+O\left(\frac{L(r)}{r}\right),
\end{aligned}
$$


or

$$
\begin{aligned}
\frac{n(r, a, w ; \Delta)}{r}+\frac{1}{2 \pi} \int_{\theta_{r}} \frac{d}{d r} \log \frac{1}{[w, a]} d \theta \\
=\frac{n(r, b, w ; \Delta)}{r}+\frac{1}{2 \pi} \int_{\theta_{r}} \frac{d}{d r} \log \frac{1}{[w, b]} d \theta+O\left(\frac{L(r)}{r}\right) .
\end{aligned}
$$

Let $d \omega(b)$ be the surface element on $K$ at $b$, then since $\pi \delta_{1}^{2}$ is the area of $\left[w, w_{0}\right]<\delta_{1}$ on $K$, taking the integral mean over $\left[w, w_{0}\right] \leqq \delta_{1}<\delta$, we have

$$
\begin{aligned}
\frac{n(r, a, w ; \Delta)}{r} & +\frac{1}{2 \pi} \int_{\theta_{r}} \frac{d}{d r} \log \frac{1}{[w, a]} d \theta=\frac{S_{1}(r, w ; \Delta)}{r} \\
& +\frac{1}{2 \pi^{2} \delta_{1}^{2}} \int_{\theta_{r}} d \theta \int_{\left[b, w_{0}\right] \leq \delta_{1}} \frac{d}{d r} \log \frac{1}{[w, b]} d \omega(b)+O\left(\frac{L(r)}{r}\right),
\end{aligned}
$$

where $S_{1}(r, w ; \Delta)=\frac{A_{1}(r, w ; \Delta)}{\pi \delta_{1}^{2}}, A_{1}(r, w ; \Delta)$ being the total area on $K$ over $\left[w, w_{0}\right] \leqq \delta_{1}$, which is covered by $w=w(z)$, when $z$ varies in $\Delta_{r}$.

By Ahlfors' first covering theorem $\left({ }^{3}\right)$,

$$
S(r, w ; \Delta)-S_{1}(r, w ; \Delta)=O(L(r))
$$

hence

$$
\begin{aligned}
\frac{n(r, a, w ; \Delta)}{r} & +\frac{1}{2 \pi} \int_{\theta_{r}} \log \frac{1}{[w, a]} d \theta=\frac{S(r, w ; \Delta)}{r} \\
& +\frac{1}{2 \pi^{2} \delta_{1}^{2}} \int_{\theta_{r}} d \theta \int_{\left[b, w_{0}\right] \leq \delta_{1}} \frac{d}{d r} \log \frac{1}{[w, b]} d \omega(b)+O\left(\frac{L(r)}{r}\right) .
\end{aligned}
$$

Similarly for $U(w)$,

$$
\begin{array}{r}
\frac{n(r, U(a), U(w) ; \Delta)}{r}+\frac{1}{2 \pi} \int_{\theta_{r}} \frac{d}{d r} \log \frac{1}{[U(w), U(a)]} d \theta=\frac{S(r, U(w) ;\lrcorner)}{r} \\
+\frac{1}{2 \pi^{2} \delta_{1}^{2}} \int_{\theta_{r}} d \theta \int_{\left[b, w_{0}\right] \leq \delta_{1}} \frac{d}{d r} \log \frac{1}{[U(w), b]} d \omega(b)+O\left(\frac{L(r)}{r}\right) .
\end{array}
$$

(3) L. AhlFoRs: Zur Theorie der Überlagerungsflächen. Acta. Math. 65 (1935).. 
Since $n(r, a, w ; d)=n(r, U(a), U(w) ; \Delta)$, we have from (20), (21),

$$
\begin{aligned}
\frac{1}{2 \pi} \int_{\theta_{r}} \frac{d}{d r} \log \frac{[U(w), U(a)]}{[w, a]} d \theta=\frac{S(r, w ; \Delta)-S(r, U(w) ; \Delta)}{r} \\
+\frac{1}{2 \pi^{2} \delta_{1}^{2}} \int_{\theta_{r}} d \theta \int_{\left[b, w_{0}\right] \leq \delta_{1}} \frac{d}{d r} \log \frac{[U(w), b]}{[w, b]} d \omega(b)+O\left(\frac{L(r)}{r}\right) .
\end{aligned}
$$

Since $L(r)=r \int_{\theta_{r}} \frac{\left|w^{\prime}\right|}{1+|w|^{2}} d \theta$, we have by the Lemma,

$$
S(r, w ; \Delta)-S(r, U(w) ; \Delta)=O(L(r)), \quad \text { q. e. d. }
$$

\section{Proof of Theorem I.}

Let $\theta_{r}$ consist of a certain number of circular ares on $\left|z-z_{0}\right|=r$, whose end points are $r e^{i \theta_{1}(r)}, r e^{i_{2}(r)}\left(\theta_{2}(r)>\theta_{1}(r)\right)$ and put $\theta(r)=\sum\left(\theta_{2}(r)-\theta_{1}(r)\right)$. We put $w_{1}=w\left(r e^{i \theta_{1}}\right), w_{2}=w\left(r e^{i \theta_{2}}\right)$, then $w_{1}, w_{2}$ lie on $\left[w, w_{0}\right]=\delta$, so that $\left[w_{1}, w_{0}\right]=\left[w_{2}, w_{0}\right]=\delta$.

Let $a$ be a point in $\left[w, w_{0}\right] \leqq \delta_{1}<\delta$, then by (17),

$$
\begin{gathered}
\frac{d}{d r} m\left(r, w_{0}, w ; \Delta\right)-\frac{d}{d r} m(r, a, w ; \Delta)=\frac{1}{2 \pi} \frac{d}{d r} \int_{\theta_{r}} \log \frac{[w, a]}{\left[w, w_{0}\right]} d \theta= \\
\frac{1}{2 \pi} \sum\left(\log \frac{\left[w_{2}, a\right]}{\left[w_{2}, w_{0}\right]} \frac{d \theta_{2}}{d r}-\log \frac{\left[w_{1}, a\right]}{\left[w_{1}, w_{0}\right]} \frac{d \theta_{1}}{d r}\right)+\frac{1}{2 \pi} \int_{\theta_{r}} \frac{d}{d r} \log \frac{[w, a]}{\left[w, w_{0}\right]} d \theta \\
=\frac{1}{2 \pi} \sum\left(\log \frac{\left[w_{2}, a\right]}{\delta} \frac{d \theta_{2}}{d r}-\log \frac{\left[w_{1}, a\right]}{\delta} \frac{d \theta_{1}}{d r}\right)+ \\
+\frac{n(r, a, w ; \Delta)-n\left(r, w_{0}, w ; \Delta\right)}{r}+O\left(\frac{L(r)}{r}\right)
\end{gathered}
$$

Hence

$$
\begin{aligned}
& T\left(r, w_{\theta}, w ; \Delta\right)=T(r, a, w ; \Delta)+ \\
& \quad+\frac{1}{2 \pi} \int_{r_{0}}^{r} \sum\left(\log \frac{\left[w_{2}, a\right]}{\delta} \frac{d \theta_{2}}{d r}-\log \frac{\left[w_{1}, a\right]}{\delta} \frac{d \theta_{1}}{d r}\right) d r+O\left(\int_{r_{0}}^{r} \frac{L(r)}{r} d r\right) .
\end{aligned}
$$

we have

Multiplying $d \omega(a)$ and taking the integral mean over $\left[w, w_{0}\right] \leqq \delta_{1}<\delta$, 


$$
\begin{gathered}
T\left(r, w_{0}, w ; \Delta\right)=\frac{1}{\pi \delta_{1}^{2}} \int_{\left[a_{0} w_{0}\right] \leq \delta_{1}} T(r, a, w ; \Delta) d \omega(a) \\
+\frac{1}{2 \pi^{2} \delta_{1}^{2}} \int_{r_{0}}^{r}\left(\int_{\left[a_{0}, w_{0}\right] \leq \delta_{1}}\left(\log \frac{\left[w_{2}, a\right]}{\delta} \frac{d \theta_{2}}{d r}-\log \frac{\left[w_{1}, a\right]}{\delta} \frac{d \theta_{1}}{d r}\right) d \omega(a)\right) d r \\
+O\left(\int_{r_{0}}^{r} \frac{L(r)}{r} d r\right) .
\end{gathered}
$$

Now by (19),

$$
\begin{aligned}
\frac{1}{\pi \delta_{1}^{2}} \int_{\left[a, w_{0}\right] \leq \delta_{1}} T(r, a, w ; \Delta) d \omega(a) & =\int_{r_{0}}^{r} \frac{S_{1}(r, w ; \Delta)}{r} d r+O(1) \\
& =\int_{r_{0}}^{r} \frac{S(r, w ; \Delta)}{r} d r+O\left(\int_{r_{0}}^{r} \frac{L(r)}{r}\right) .
\end{aligned}
$$

Since, $w_{1}, w_{2}$ lying on $\left[w, w_{0}\right]=\delta$,

$$
\frac{1}{2 \pi^{2} \delta_{1}^{2}} \int_{\left[a, w_{0}\right] \leq \delta_{1}} \log \frac{\left[w_{1}, a\right]}{\delta} d \omega(a)=\frac{1}{2 \pi^{2} \delta_{1}^{2}} \int_{\left[a_{0} w_{0}\right] \leq \delta_{1}} \log \frac{\left[w_{2}, a\right]}{\delta} d \omega(a)=A=\text { const. }
$$

the second term of (24) becomes

$$
A \int_{r_{0}}^{r} \frac{d}{d r} \Sigma\left(\theta_{2}(r)-\theta_{1}(r)\right) d r=A \int_{r_{0}}^{r} \frac{d \theta(r)}{d r} d r=A\left(\theta(r)-\theta\left(r_{0}\right)\right)=O(1)
$$

Hence from (24), (25), (26), we have

$$
T\left(r, w_{0}, w ; \Delta\right)=\int_{r_{0}}^{r} \frac{S(r, w ; \Delta)}{r} d r+O\left(\int_{r_{0}}^{r} \frac{L(r)}{r} d r\right) .
$$

Let $a$ be any point in $\left[w, w_{0}\right]<\delta$ and $U(w)$ be the linear transformation, which makes $\left[w, w_{0}\right]<\delta$ invariant and bring $a$ to $w_{0}$, so that $w_{0}=U(a)$, then

$$
\begin{aligned}
& T\left(r, w_{0}, U(w) ; \Delta\right)=T(r, U(a), U(w) ; \Delta) \\
&=N(r, U(a), U(w) ; \Delta)+\frac{1}{2 \pi} \int_{\theta_{r}} \log \frac{1}{[U(w), U(a)]} d \theta= \\
& N(r, a, w ; \Delta)+\frac{1}{2 \pi} \int_{\theta_{r}} \log \frac{1}{[w, a]} d \theta+O(1)=T(r, a, w ; \Delta)+O(1) .
\end{aligned}
$$


Hence from (27)

$$
\begin{aligned}
& T(r, a, w ; \Delta)=T\left(r, w_{0},\right.U(w) ; \Delta)+O(1) \\
&=\int_{r_{0}}^{r} \frac{S(r, U(w) ; \Delta)}{r} d r+O\left(\int_{r_{0}}^{r} \frac{L_{1}(r)}{r} d r\right),
\end{aligned}
$$

where $L_{1}(r)$ is the length of the image of $\theta_{r}$ on $K$ by $w=U(w(z))$. Since $L_{1}(r)=O(L(r))$, we have by Theorem II,

$$
T(r, a, w ; \Delta)=\int_{r_{0}}^{r} \frac{S(r ; w ; \Delta)}{r} d r+O\left(\int_{r_{0}}^{r} \frac{L(r)}{r} d r\right),
$$

which proves the first part.

$$
\text { Since }, A(r)=\int_{r_{0}}^{r} \int_{\theta_{r}} \frac{\left|w^{\prime}\right|^{2}}{\left(1+|w|^{2}\right)^{2}} r d r d \theta, \quad L(r)=r \int_{\theta_{r}} \frac{\left|w^{\prime}\right|}{1+|w|^{2}} d \theta,
$$

we have

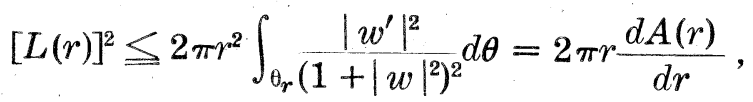

so that

$$
\int_{r_{0}}^{r} \frac{L(r)}{r} d r \leqq \sqrt{\log r \int_{r_{0}}^{r} \frac{[L(r)]^{2}}{r}} d r \leqq \sqrt{2 \pi \log r \cdot A(r)}=O(\sqrt{T(2 r) \log r)}
$$

which proves (2).

Remark. Instead of $\left[w, w_{0}\right]<\delta$, we consider a domain $D$ on $K$, which is bounded by an analytic Jordan curve. Let $w=w(z)$ be meromorphic in $\Delta$ and takes in $\Delta$ the values which belong to $D$ and the bonndary of $\Delta$ corresponds to the boundary of $D$. We define $T(r, a, w ; \Delta)$ etc as in $\$ 1$. We map $D$ conformally on $\left[v, v_{0}\right]<\delta$ by $w=\psi(v)$ and let $w=w(z)$ become $v=v(z)$. Let $L(r), L_{1}(r)$ be the length of the images of $\theta_{r}$ by $w=w(z)$ and $v=v(z)$ on $K$, then $L_{1}(r)=O(L(r))$. By Theorem I, for any two points $\alpha, \beta$ in $\left[v, v_{0}\right]<\delta$,

$$
\begin{aligned}
T(r, \alpha, v ; \Delta) & =T(r, \beta, v ; \Delta)+O\left(\int_{r_{0}}^{r} \frac{L_{1}(r)}{r} d r\right) \\
& =T(r, \beta, v ; \Delta)+O\left(\int_{r_{0}}^{r} \frac{L(r)}{r} d r\right) .
\end{aligned}
$$


Since $T(r, a, v ; \Delta)=T(r, a, w ; d)+O(1)$, where $a=\psi(\alpha)$, we have for any two points $a, b$ in $D_{1}<D$,

$$
T(r, a, w ; \Delta)=T(r, b, w ; \Delta)+O\left(\int_{r_{0}}^{r} \frac{L(r)}{r} d r\right) .
$$

Multiplying $d \omega(b)$ and taking the integral mean over $D_{1}<D$, we have by (19)

$$
T(r, a, w ; \Delta)=\int_{r_{0}}^{r} \frac{S(r, w ; \Delta)}{r} d r+O\left(\int_{r_{0}}^{r} \frac{L(r)}{r} d r\right) .
$$

Hence Theorem I holds, if $\left[w, w_{0}\right]<\delta$ is replaced by any domain $D$ on $K$ bounded by an analytic Jordan curve.

\section{Theorems on $T(r)$.}

We will prove some theorems on $T(r)$.

Theorem III. For any point $a$ in $\left[w, w_{0}\right]<\delta$,

(i ) $\varlimsup_{r \rightarrow \infty} \frac{\log T(r)}{\log r}=\varlimsup_{r \rightarrow \infty} \frac{\log T(r, a)}{\log r}$,

(ii) $\varlimsup_{r \rightarrow \infty} \frac{T(r)}{r^{\rho}}=\varlimsup_{r \rightarrow \infty} \frac{T(r, a)}{r^{p}}, \quad(\rho>0)$,

(iii) $\int_{r_{0}}^{\infty} \frac{T(r)}{r^{\rho+1}} d r$ and $\int_{r_{0}}^{\infty} \frac{T(r, a)}{r^{\rho+1}} d r(\rho>0)$ are finite or infinite at the : same time. $\left({ }^{4}\right)$

Proof. (i) We put $\varlimsup_{r \rightarrow \infty} \frac{\log T(r)}{\log r}=\rho, \quad \varlimsup_{r \rightarrow \infty} \frac{\log T(r, a)}{\log r}=\rho_{1}$.

(a) If $\rho<\infty$, then $\rho=\rho_{1}$ follows from $\left(1^{*}\right)$.

(b) If $\rho_{1}<\infty$, then from (1), (3), we have outside $I_{n}=\left(r_{1}^{(n)}, r_{2}^{(n)}\right)$, $T(r, a)=T(r)+O(\sqrt{T(r)} \log T(r)) \geqq(1-\varepsilon) T(r)$ or $T(r) \leqq r^{\rho_{1}+\varepsilon}$ for $r \geqq r_{0}$

(4) c. f. K. Kunugur: Sur la théorie des fonctions méromorphes et uniformes. Jap. Journ. Math. 18 (1942). 
Especially $T\left(r_{2}^{(n)}\right) \leqq\left[r_{2}^{(n)}\right]^{\rho_{1}+\varepsilon} \quad$ Since $\sum_{n} \int d \log \log r<\infty, r_{2}^{(n)} \leqq\left[r_{1}^{(n)}\right]^{K}$ $(K=$ const. $(>1))$. Hence for $r_{1}^{(n)} \leqq r \leqq r_{2}^{(n)}$,

$$
T(r) \leqq T\left(r_{2}^{(n)}\right) \leqq\left[r_{2}^{(n)}\right]^{\rho_{1}^{+}+\varepsilon} \leqq\left[r_{1}^{(n)}\right]^{K\left(p_{1}+\varepsilon\right)} \leqq r^{K\left(\rho_{1}+\varepsilon\right)} .
$$

Consequently we have from (30), $T(r) \leqq r^{K\left(\rho_{1}+\varepsilon\right)}$ for all $r \geqq r_{0}$, so that $\rho \leqq K \rho_{1}<\infty$. Hence if $\rho=\infty$, then $\rho_{1}=\infty$.

From (a), (b), we have $\rho=\rho_{1}$ always, the both sides being finite or infinite.

(ii) We put $\varlimsup_{r \rightarrow \infty} \frac{T(r)}{r^{\rho}}=\Lambda, \varlimsup_{r \rightarrow \infty} \frac{T(r, a)}{r^{\rho}}=\Lambda_{1}$.

(a) If $\Lambda<\infty$, then $A=\Lambda_{1}$ from $\left(1^{*}\right)$.

(b) If $\Lambda_{1}<\infty$, then by (i), $T(r)=O\left(r^{p+\varepsilon}\right)$, so that $\Lambda=\Lambda_{1}$ from $\left(1^{*}\right)$. Hence if $\Lambda=\infty$, then $\Lambda_{1}=\infty$.

From (a), (b), we have $\Lambda=\Lambda_{1}$ always, the both sides being finite or infinite.

(iii) (a) If $\int_{r_{0}}^{\infty} \frac{T(r)}{r^{p+1}} d r<\infty$, then $T(r)=O\left(r^{\rho}\right)$, hence $\int_{r_{0}}^{\infty} \frac{T(r, a)}{r^{p+1}} d r<\infty$ from $\left(1^{*}\right)$.

(b) If $\int_{r_{0}}^{\infty} \frac{T(r, a)}{\rho^{\rho+1}} d r<\infty$, then $T(r, a)=O\left(r^{\rho}\right)$, so that by (i), $T(r)=O\left(r^{\rho+\varepsilon}\right)$, hence $\int_{r_{0}}^{r_{0}} \frac{T(r)}{r^{\rho+1}} d r<\infty$. from $\left(1^{*}\right)$.

From (a), (b), $\int_{r_{0}}^{\infty} \frac{T(r)}{r^{\rho+1}} d r$ and $\int_{r_{0}}^{\infty} \frac{T(r, a)}{r^{\rho+1}} d r$ are finite or infinite at the same time.

Theorem IV. Let $f(z)$ be regular in $\Delta$, where $|f(z)|>\lambda$ in $\Delta$ and $|f(z)|=\lambda$ on the boundary of $\Delta$ and $M(r)=\operatorname{Max}_{\theta_{r}}|f(z)|$. Then

(i) $\varlimsup_{r \rightarrow \infty} \frac{\log T(r)}{\log r}=\varlimsup_{r \rightarrow \infty} \frac{\log \log M(r)}{\log r}$,

(ii) $\varlimsup_{r \rightarrow \infty} \frac{T(r)}{r^{\rho}}$ and $\varlimsup_{r \rightarrow \infty} \frac{\log M(r)}{r^{p}}(\rho>0)$ are zero, positive or infinite at the same time.

(iii) $\int_{r_{0}}^{\infty} \frac{T(r)}{r^{p+1}} d r$ and $\int_{r_{0}}^{\infty} \frac{\log M(r)}{r^{p+1}} d r(\rho>0)$ are finite or infinite at the same time $\left({ }^{4}\right)$. 
Proof. (i) We put $\varlimsup_{r \rightarrow \infty} \frac{\log T(r)}{\log r}=\rho, \quad \varlimsup_{r \rightarrow \infty} \frac{\log \log M(r)}{\log r}=\rho_{1}$.

(a) If $\rho<\infty$, then $\log M(r) \leqq 3 m(2 r, \infty)+\operatorname{const.}\left({ }^{5}\right)$, so that by $\left(1^{*}\right)$,

$$
\log M(r) \leqq 3 T(2 r, \infty)+\text { const. }=3 T(2 r)+O\left(r^{\rho^{\prime}}\right)\left(\rho^{\prime}<\rho\right) .
$$

Hence $\rho_{1} \leqq \rho$. From $\left(1^{*}\right)$,

$$
T(r)=T(r, \infty)+O\left(r^{\rho^{\prime}}\right)=m(r, \infty)+O\left(r^{\rho^{\prime}}\right) \leqq \log M(r)+O\left(r^{\rho^{\prime}}\right) \quad\left(\rho^{\prime}<\rho\right) .
$$

Hence $\rho \leqq \rho_{1}$, so that $\rho=\rho_{1}$.

(b) If $\rho_{1}<\infty$, then by (1), (3) we have outside $I_{n}$ for $r>r_{0}$,

$$
\begin{aligned}
(1-\varepsilon) T(r) \leqq T(r)+O(\sqrt{T(r)} \log T(r)) & =T(r, \infty) \\
& =m(r, \infty) \leqq \log M(r)+O(1),
\end{aligned}
$$

so that $T(r)<r^{p_{1}+\varepsilon}$. Similarly as in the proof of Theorem III (i), (b), we have $\rho \leqq K \rho_{1}<\infty$. Hence if $\rho=\infty$, then $\rho_{1}=\infty$. infinite.

From $(a)$, (b) we have $\rho=\rho_{1}$ always, the both sides being finite or

(ii) We put $\varlimsup_{r \rightarrow \infty} \frac{T(r)}{r^{\rho}}=\Lambda, \quad \varlimsup_{r \rightarrow \infty} \frac{\log M(r)}{r^{p}}=\Lambda_{1}$.

(a) If $\Lambda=0$, then $\Lambda_{1}=0$ from (31).

(b) If $0<\Lambda<\infty$, then $\Lambda \leqq \Lambda_{1} \leqq 3.2^{p} \Lambda$ from (31), (32).

(c) If $\Lambda=\infty$, then $\Lambda_{1}=\infty$. For, if $\Lambda_{1}<\infty$, then $T(r)=O\left(r^{p+\varepsilon}\right)$ by (i). Hence $\Lambda \leqq \Lambda_{1}<\infty$ from (32), which contradicts the hypothesis. Hence $\Lambda_{1}=\infty$.

(iii) (a) If $\int_{r_{0}}^{\infty} \frac{T(r)}{r^{\rho+1}} d r<\infty$, then $\int_{r_{0}}^{\infty} \frac{\log M(r)}{r^{\rho+1}} d r<\infty$ from (31).

(b) If $\int_{r_{0}}^{\infty} \frac{\log M(r)}{r^{p+1}} d r<\infty$, then $\log M(r)=O\left(r^{\rho}\right)$, so that $T(r)=O\left(r^{p+\varepsilon}\right)$ by (i), hence $\int_{r_{0}}^{\infty} \frac{T(r)}{r^{\beta+1}} d r<\infty$ from (32).

(5) Nevanlinna: Eindeutige analytische Funktionen. p. 294. 
From (a), (b), $\int_{r_{0}}^{\infty} \frac{T(r)}{r^{\rho+1}} d r$ and $\int_{r_{0}}^{\infty} \frac{\log M(r)}{r^{\rho+1}} d r$ are finite or infinite at the same time.

Theorem V. Let $U(w)$ be a linear transformation, which makes $\left[w, w_{0}\right]<\delta$ invariant. Then

$$
\begin{aligned}
& T(r, U(w) ; \Delta)=T(r, w ; \Delta)+O\left(\int_{r_{0}}^{r} \frac{L(r)}{r} d r\right), \\
& \varlimsup_{r \rightarrow \infty} \frac{\log T(r, w ; \Delta)}{\log r}=\varlimsup_{r \rightarrow \infty} \frac{\log T(r, U(w) ; \Delta)}{\log r} .
\end{aligned}
$$

Proof. (i) follows from Theorem II.

(ii) We put $\varlimsup_{r \rightarrow \infty} \frac{\log T(r, w ; \Delta)}{\log r}=\rho, \varlimsup_{r \rightarrow \infty} \frac{\log T(r, U(w) ; \Delta)}{\log r}=\rho_{1}$.

If $\rho<\infty$, then from (i) and (2),

$$
T(r, U(w) ; \Delta)=T(r, w ; \Delta)+O\left(r^{\rho^{\prime}}\right) \quad\left(\rho^{\prime}<\rho\right) .
$$

Hence $\rho=\rho_{1}$. Similarly $\rho=\rho_{1}$ if $\rho_{1}<\infty$, q. e. d.

If $\left[w, w_{0}\right]<\delta$ contains a transcendental singularity $a$ of the inverse function $\phi(w)$ of $w=f(z)$, then it is proved by K. Noshiro $\left({ }^{6}\right)$ and K. Kunugui $\left({ }^{7}\right)$ that $\lim _{r \rightarrow \infty} A(r)=\infty$. We will give a simple proof for it.

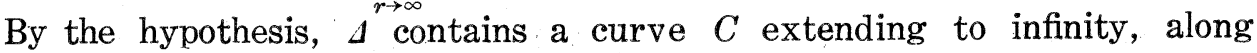
which $f(z)$ tends to $a$. Let $\gamma$ be the image of $C$ on $K$, then $\gamma$ ends at a. There are two cases.

(i) $\Delta$ contains a boundary curve which extends to infinity.

In this case, an arc $a_{r}$ of $\theta_{r}$ meets $C$ and the boundary of $\Delta$, so that the image of $\alpha_{r}$ on $K$ meets $\left[w, w_{0}\right]=\delta$ and $\gamma$. Hence the length of this image has a length $\geqq d>0$ for $r>r_{0}$, so that $L(r) \geqq d>0$ for $r>r_{0}$. From (29), we have

$$
d^{2} \log \frac{r}{r_{0}} \leqq \int_{r_{0}}^{r} \frac{[L(r)]^{2}}{r} d r \leqq 2 \pi A(r),
$$

hence $\lim _{r \rightarrow \infty} A(r)=\infty$.

(ii) $\Delta$ contains no boundary curve which extends to infinity.

Then the boundary of $\Delta$ consists of infinitely many closed curves $17(1940)$.

(6) K. Noshiro: On the singularities of analytic functions. Jap. Journ. Math.

( 7 ) K. KUNUGUI : 1. c. (4). 
clustering to infinity. For, if there are only finite number of closed boundary curves, then $|z|>r_{0}$ belongs to $\Delta$, so that $f(z)$ is meromorphic or has an essential singularity at $z=\infty$. By the hypothesis, $f(z)$ is not meromorphic at $z=\infty$. If $f(z)$ has an essential singularity at $z=\infty$, then by Picard's theorem, $f(z)$ takes the values outside $\left[w, w_{0}\right]<\delta$, which contradicts the ypothesis. Hence there are infinitely many closed boundary curves clustering to infinity. In this case we have two cases.

Case (a). $\phi(w)$ ha: no transcendental singularity other than $a$ in $\left[w, w_{0}\right]<\delta$. Then starting from any element of $\phi(w)$, we can continuate $\varphi(w)$ analytically along any curve which does not pass $a$. Then we see easily that if $f(z)-b(b \neq a)$ has a finite number of zero points in $\Delta, n$, say, then $f(z)-w$ has $n$ zero points in $\Delta$ for any $w(\neq a)$ in $\left[w, w_{0}\right]<\delta$. Consequently there are at most $n$ closed boundary curves, which is a contradiction. Hence $f(z)-w$ has infinitely zero points for any $w(\neq a)$ in $\left[w, w_{0}\right]<\delta$. Hence $\lim _{r \rightarrow \infty} A(r)=\infty$.

Case (b). $\phi(w)$ has a transcendental singularity $b(\neq a)$ in $\left[w, w_{0}\right]<\delta$. Then there exists a curve $C^{\prime}$ extending to infinity along which $f(z)$ tends to $b$, so that its image $\gamma^{\prime}$ on $K$ ends at $b$. Then an arc $\alpha_{r}$ of $\theta_{r}$ which meets $C$, meets the boundary of $\Delta$ or $C^{\prime}$. In each case, the image of $a_{r}$ on $K$ has a length $\geqq d>0$ for $r>r_{0}$, so that $L(r) \geqq d>0$ for $r>r_{0}$. Whence as before we conclude that $\lim _{r \rightarrow \infty} A(r)=\infty$.

Since

$$
\lim _{r \rightarrow \infty} S(r)=\lim _{r \rightarrow \infty} \frac{A(r)}{\pi \delta^{2}}=\infty, \text { we have }
$$

Theorem VI. If $\left[w, w_{0}\right]<\delta$ contains a transcendental singularity of the inverse function $\phi(w)$ of $w=f(z)$, then

$$
\lim _{r \rightarrow \infty} \frac{T(r)}{\log r}=\infty \text {. }
$$

\section{Second Fundamental Theorem.}

Let $D_{1}, \ldots, D_{q}(q \geqq 2)$ be $q$ simply connected domains in $\left[w, w_{0}\right]<\delta$, which have no common points with each other and $D$ be any one of $D_{i}$.

Let $F_{r}$ be the image of $\Delta_{r}$ on $K$ by $w=f(z)$. The part of $F_{r}$ which lies above $\mathrm{D}$ consists of a certain number of connected pieces which are of two types. Those pieces which have no relative boundaries in $D$ are called islands and the other pieces are called peninsulas. We apply Ahlfors' theory of covering surfaces $\left({ }^{8}\right)$ to $F_{r}$.

We denote the circular disc $\left[w, w_{0}\right] \leqq \delta$ by $F_{0}$ and the Euler's characteristic of a-domain $D$ by $\rho(D)$ and $p(D)=-\rho(D)$ is called the simple

(8) Ahlfors; 1. c. (3). 
multiplicity of $D$, which is +1 , if $D$ is simply connected, otherwise $p(D) \leqq 0$.

We denote the sum of the simple multiplicities of all islands $D^{i}$ of $F_{r}$ above $D_{k}$ by $\bar{p}\left(D_{l c}\right)$.

First we take off from $F_{r}$ all the peninsulas above $D_{1}+\cdots+D_{q}$, then there remains a certain number of connected pieces $\sum F_{r}^{\prime}$.

From $\sum F_{r}^{\prime}$ we take off all the islands $D^{i}$ above $D_{1}+\cdots+D_{q}$, then there remains a certain number of connected pieces $\sum \bar{F}_{r}$, so that

Hence

$$
\sum F_{r}^{\prime}=\sum D^{\boldsymbol{i}}+\sum \bar{F}_{r} .
$$

$$
\sum \rho\left(F_{r}^{\prime}\right)=\sum \rho\left(D^{i}\right)+\sum \rho\left(\bar{F}_{r}\right) .
$$

Considering on the $z$-plane, we have easily

$$
\begin{aligned}
\sum_{k=1}^{q} \bar{p}\left(D_{k}\right)=\sum \rho\left(\bar{F}_{r}\right)-\sum \rho\left(F_{r}^{\prime}\right) & =\sum \rho^{+}\left(\bar{F}_{r}\right)-\left(\sum \rho\left(F_{r}^{\prime}\right)+N_{1}\left(\bar{F}_{r}\right)\right) \\
& \geqq \sum \rho^{+}\left(\bar{F}_{r}\right)-\sum \rho^{+}\left(F_{r}^{\prime}\right),
\end{aligned}
$$

where $N_{1}\left(\bar{F}_{r}\right)$ is the number of simply connected pieces in $\sum \bar{F}_{r}$.

We put $\bar{F}_{0}=F_{0}-\left(D_{1}+\cdots+D_{q}\right)$. By Ahlfors' fundamental theorem,

$$
\sum \rho^{+}\left(\bar{F}_{r}\right) \geqq(q-1) S\left(\bar{F}_{0}\right)-h L(r),
$$

where $h$ is a constant and $S\left(\bar{F}_{0}\right)=\frac{\text { area of } \sum \bar{F}_{r}}{\text { area of } \bar{F}_{0}}$ and by Ahlfors' first covering theorem, $S\left(\bar{F}_{0}\right) \geqq S(r)-h L(r)$, so that

$$
\sum \rho^{+}\left(\bar{F}_{r}\right) \geqq(q-1) S(r)-h L(r),
$$

where $S(r), L(r)$ have the same meanings as in $\S 1$.

Considering on the $z$-plane, we see easily

$$
\sum \rho^{+}\left(F_{r}^{\prime}\right) \leqq \lambda(r),
$$

where

$$
\lambda(r)=\text { the number of holes in } \Delta_{r} .
$$

Hence

$$
\sum_{k=1}^{q} \bar{p}\left(D_{k}\right) \geqq(q-1) S(r)-\lambda(r)-h L(r) .
$$

We take $a_{k}$ in $D_{k}$, then, since $\bar{p}\left(D_{k}\right) \leqq n\left(r, \dot{a}_{k}\right)$, we have

$$
(q-1) S(r) \leqq \sum_{k=1}^{q} n\left(r, a_{k}\right)+\lambda(r)+h L(r) .
$$


Next we will evaluate $\lambda(r)$. Now $F_{r}$ consists of a ceratin number of sheets and let $\Omega_{i}$ be such a sheet whose boundary consists of the whole circle $\left[w, w_{0}\right]=\delta$ and one part of the image of $\theta_{r}$, which form holes in $\Omega_{i}$. Let the sum of the lengths of the boundaries of the holes of $\Omega_{i}$ be denoted by $L_{i}$, then $\mid$ area of $\Omega_{i}-\pi \delta^{2} \mid \leqq h L_{i}$, where $h$ is a constant. Hence summing up for such $\Omega_{i}$, we have

$$
S(r) \geqq \frac{\sum \text { area of } \Omega_{i}}{\pi \delta^{2}} \geqq \lambda(r)-h L(r)
$$

Hence

$$
(q-1) S(r) \leqq \sum_{k=1}^{q} n\left(r, a_{k}\right)+\lambda(r)+h L(r)
$$

where

$$
\lambda(r) \leqq S(r)+h L(r) .
$$

From (39), (40), we have the following theorem, which corresponds to Nevanlinna's second fundamental theorem.

Theorem VII (SECOND FUNDAMENTAL THEOREM). For any $q$ points, $a_{1}, \ldots, a_{q}$ in $\left[w, w_{0}\right]<\delta$,

$$
(q-1) T(r) \leqq \sum_{k=1}^{q} N\left(r, a_{k}\right)+\Lambda(r)+O\left(\int_{r_{0}}^{r} \frac{L(r)}{r} d r\right)
$$

where

$$
\Lambda(r)=\int_{r_{0}}^{r} \frac{\lambda(r)}{r} d r \leqq T(r)+O\left(\int_{r_{0}}^{r} \frac{L(r)}{r} d r\right),
$$

$\lambda(r)$ being the number of holes in $\Delta_{r}$.

\section{Some consequences of the Second Fundamental Theorem.}

(A) Let $f(z)$ take $q$ values, $a_{1}, \ldots, a_{q}$ in $\left[w, w_{0}\right]<\delta$ finite times in $\checkmark$, then from (37), (40),

$$
(q-1) S(r) \leqq \lambda(r)+h L(r)+O(1) \leqq S(r)+h L(r)+O(1)
$$

We suppose that $\left[w, w_{0}\right]<\delta$ contains a transcendental singularity of the inverse function $\varphi(w)$ of $w=f(z)$, then by Theorem VI, $\lim _{r \rightarrow \infty} S(r)=\infty$.

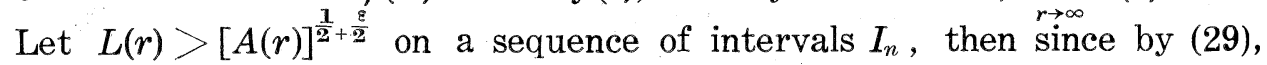
$[L(r)]^{2} \leqq 2 \pi r \frac{d A(r)}{d r}$, we have

$$
\sum_{n} \int_{I_{n}} d \log r=\sum_{n} \int_{I_{n}} \frac{d r}{r}<2 \pi \sum_{n} \int_{I_{n}} \frac{d A(r)}{[A(r)]^{1+\varepsilon}}<2 \pi \int_{r_{0}}^{\infty} \frac{d A}{A^{1+\varepsilon}}<\infty .
$$

Since outside $I_{n}, L(r) \leqq[A(r)]^{\frac{1}{2}+\frac{1}{2} \varepsilon}=\left[\pi \delta^{2} S(r)\right]^{\frac{1}{2}+\frac{1}{2} \varepsilon}$, we have 


$$
\lim _{r \rightarrow \infty} \frac{L(r)}{S(r)}=0,
$$

where $r$ tends to infinity by taking the values outside $I_{n}$. Hence we have from (43), $q \leqq 1+\lim _{r \rightarrow \infty} \frac{\lambda(r)}{S(r)} \leqq 2$.

Theorem VIII. If $\left[w, w_{0}\right]<\delta$ contains a transcendental singularity of the inverse function $\phi(w)$ of $w=f(z)$, then $f(z)$ txkes any value $a$ in $\left[w, w_{0}\right]<\delta$, except at most $q \leqq 1+\lim _{r \rightarrow \infty} \frac{\lambda(r)}{S(r)} \leqq 2$ values, infinitely many times in $\Delta$, where $r$ tends to infinity by taking the values outside certain intervals $I_{n}$, such that $\sum_{n} \int_{I_{n}} d \log r<\infty$.

K. Noshiro $\left({ }^{9}\right)$ proved that $q \leqq 1$, if $\Delta$ is simply connected and K. Kunuguil ${ }^{10}$ ) proved that $q \leqq 2$. The general case is due to Y. Tumura. $\left({ }^{11}\right)$.

(B) Defect relation.

By (1), (3) we have outside $I_{n}, N(r, a) \leqq T(r)+O(\sqrt{T(r)} \log T(r))$, so that $\left(\varlimsup_{r \rightarrow \infty} \frac{N(r, a)}{T(r)} \leqq 1\right.$ and by (41), (42), (3), we have outside $I_{n}$,

$$
\begin{aligned}
&(q-1) T(r) \leqq \sum_{k=1}^{q} N\left(r, a_{k}\right)+\Lambda(r)+O(\sqrt{T(r)} \log T(r)) \\
& \leqq \sum_{k=1}^{q} N\left(r, a_{k}\right)+T(r)+O(\sqrt{T(r)} \log T(r)),
\end{aligned}
$$

so that

$$
(q-1) \leqq \sum_{k=1}^{q} \varlimsup_{r \rightarrow \infty} \frac{N\left(r, a_{k}\right)}{T(r)}+\lim _{r \rightarrow \infty} \frac{\Lambda(r)}{T(r)} \leqq \sum_{k=1}^{q} \varlimsup_{r \rightarrow \infty} \frac{N\left(r, a_{k}\right)}{T(r)}+1 .
$$

We will call

$$
\delta(a)=1-\varlimsup_{r \rightarrow \infty} \frac{N(r, a)}{T(r)} \geqq 0
$$

the defect of $a$. Then we have from (45),

Theorem IX. $\sum \delta(a) \leqq 1+\lim _{r \rightarrow \infty} \frac{A(r)}{T(r)} \leqq 2$,

(9) K. Noshiro: 1. c. (5).

(10) K. Kunugui : Une généralisation des théorèmes de MM. Picard-Nevanlinna sur les fonctions méromorphes. Proc. Imp. Acad. 17 (1914).

(11) Y. Tumura: 1. c. (1) also M. TsuJI: On the behaviour of an inverse function of a meromorphic function at its transcendental singular point, I. Proc. Imp. Acad. 17 (1941). 
where $r$ tends to infinity by taking the values outside certain intervals $I_{n}$, such that $\sum_{n} \int_{I_{n}} d \log \log r<\infty$.

\section{(C) Picard-Borel's theorem.}

Theorem X. If $\int_{r_{0}}^{\infty} \frac{N(r, a)}{r^{\rho+1}} d r<\infty(\rho>0)$ for three (two, if $\left.\int_{r_{0}}^{\infty} \frac{A(r)}{r^{\rho+1}} d r<\infty\right)$ values $a$ in $\left[w . w_{0}\right]<\delta$, then $\int_{r_{0}}^{\infty} \frac{T(r)}{r^{\rho+1}} d r<\infty$ and hence $\int_{r_{0}}^{\infty} \frac{N(r, a)}{r^{p+1}} d r<\infty$ for any value $a$ in $\left[w, w_{0}\right]<\delta$.

Proof. Let $\int_{r_{0}}^{\infty} \frac{N\left(r, a_{k}\right)}{r^{p+1}} d r<\infty \quad(k=1,2,3)$. Then from (41), (42), (3), we have outside $I_{n}=\left(r_{1}^{(n)}, r_{2}^{(n)}\right)(n=1,2, \ldots), \quad T(r) \leqq \sum_{k=1}^{3} N\left(r, a_{k}\right)$ $+O(\sqrt{T(r)} \log T(r))$, so that for a large $r, T(r) \leqq \frac{1}{1-\varepsilon} \sum_{k=1}^{3} N\left(r, a_{k}\right)$.

From $\int_{r_{0}}^{\infty} \frac{N\left(r, a_{k}\right)}{r^{p+1}} d r<\infty$, we have $N\left(r, a_{k}\right)=O\left(r^{\rho}\right)$, so that outside $I_{n}$.

$$
T(r) \leqq \text { const. } r^{p}
$$

From $\sum_{n} \int_{I_{n}} d \log \log r<\infty$, we have $r_{2}^{(n)} \leqq\left[r_{1}^{(n)}\right]^{1+\varepsilon}$, so that for $r_{1}^{(n)} \leqq r \leqq r_{2}^{(n)}$,

$$
\begin{aligned}
T(r) \leqq T\left(r_{2}^{(n)}\right) \leqq \text { const. }\left[r_{2}^{(n)}\right]^{p} & \leqq \text { const. }\left[r_{1}^{(n)}\right]^{p(+\varepsilon)} \\
& \leqq \text { const. } r^{p(1+\varepsilon)} .
\end{aligned}
$$

From (47), (48), we see that (48) holds for all sufficiently large $r$. Since $\varepsilon$ may be taken arbitrarily small, we have from $(41),(42),(2),\left(1^{*}\right)$,

$$
T(r) \leqq \sum_{k=1}^{3} N\left(r, a_{k}\right)+O\left(r^{\rho^{\prime}}\right) \quad\left(\rho^{\prime}<\rho\right)
$$

so that

$$
\int_{r_{0}}^{\infty} \frac{T(r)}{r^{p+1}} d r<\infty .
$$

If $\int_{r_{0}}^{\infty} \frac{T(r)}{r^{p+1}} d r<\infty$, then $\int_{r_{0}}^{\infty} \frac{T(r, a)}{r^{p+1}} d r<\infty$ from Theorem III. Hence $\int_{r_{0}}^{\infty} \frac{N(r, a)}{r^{\rho+1}} d r<\infty$ for any value $a$ in $\left[w, w_{0}\right]<\delta$. 
By using $T(r) \leqq \sum_{k=1}^{2} N\left(r, a_{k}\right)+A(r)+O\left(\int_{r_{0}}^{r} \frac{L(r)}{r} d r\right)$, we can prove that, if $\int_{r_{0}}^{\infty} \frac{\Lambda(r)}{r^{p+1}} d r<\infty$, then $\int_{r_{0}}^{\infty} \frac{T(r)}{r^{\rho+1}} d r<\infty$ follows from $\int_{r_{0}}^{\infty} \frac{N\left(r, a_{k}\right)}{r^{\rho+1}} d r<\infty$ $(k=1,2)$.

The following theorem corresponds to Picard-Borel's theorem.

Theorem XI. Let $f(z)$ be meromorphic and of finite order $\rho$ in $\Delta$ and $a$ be any point in $\left[w, w_{0}\right]<\delta$. Then $N(r, a)$ is of the same order (class, type) as $T(r)$, except for at most two (one, if $\varlimsup_{r \rightarrow \infty} \frac{\log \Lambda(r)}{\log r}<\rho$ ) values of $a$ in $\left[w, w_{0}\right]<\delta$, for which $N(r, a)$ may be of lower order (class, type) than $T(r)$.

Proof.

(i) We put $\rho(a)=\varlimsup_{r \rightarrow \infty} \frac{\log N(r, a)}{\log r}$, then by Theorem III,

$$
\rho(a) \leqq \varlimsup_{r \rightarrow \infty} \frac{\log T(r, a)}{\log r}=\varlimsup_{r \rightarrow \infty} \frac{\log T(r)}{\log r}=\rho .
$$

If $\rho\left(a_{k}\right)<\rho(k=1,2,3)$, then from (49), the order of $T(r)$ would be lower than $\rho$, contradictory to the hypothesis. Hence $\rho(a)=\rho$, except for at most two values $a$ in $\left[w, w_{0}\right]<\delta$.

(ii) From Theorem III, if $\int_{r_{0}}^{\infty} \frac{T(r)}{r^{p+1}} d r<\infty$, then $\int_{r_{0}}^{\infty} \frac{N(r, a)}{r^{p+1}} d r<\infty$ for every value $a$ in $\left[w, w_{0}\right]<\delta$ and if $\int_{r_{0}}^{\infty} \frac{T(r)}{r^{p+1}} d r=\infty$, then from (49) $\int_{r_{0}}^{\infty} \frac{N(r, a)}{r^{p+1}} d r=\infty$, except for at most two values $a$ in $\left[w, w_{0}\right]<\delta$.

(iii) We put $\lim _{r \rightarrow \infty} \frac{T(r)}{r^{p}}=\Lambda, \varlimsup_{r \rightarrow \infty} \frac{N(r, a)}{r^{p}}=\nu(a)$, then by Theorem III,

$$
\nu(a) \leqq \varlimsup_{r \rightarrow \infty} \frac{T(r, a)}{r^{p}}=\varlimsup_{r \rightarrow \infty} \frac{T(r)}{r^{p}}=\Lambda,
$$

and from (49),

$$
\Lambda \leqq \sum_{k=1}^{8} \nu\left(a_{k}\right)
$$

From (50), (51), we have

(a) If $\Lambda=0$, then $\nu(a)=0$ for any value $a$ in $\left[w, w_{0}\right]<\delta$.

(b) If $0<\Lambda<\infty$, then $0<\nu(a)<\infty$, except for at most two values $a$ in $\left[w, w_{0}\right]<\delta$.

(c) If $A=\infty$, then $\nu(a)=\infty$, except for at most two values $a$ in $\left[w, w_{0}\right]<\delta$. 
We can prove that, if $\varlimsup_{r \rightarrow \infty} \frac{\log A(r)}{\log r}<\rho$, then the number of exceptional values is reduced to one.

\section{(D) Discs-theorem.}

The Riemann surface $F$ of $\phi(w)$ is called $\mu$-ply ramified above $D$; if all the simply connected islands above $D$ have at least $\mu$ sheets. If $F$ ramifies $\mu_{k}$-ply above $D_{k}$, then from (36),

$$
\sum_{k=1}^{q} \frac{1}{\mu_{k}} S\left(D_{k}\right) \geqq \sum_{k=1}^{q} \bar{p}\left(D_{k}\right) \geqq(q-1) S(r)-\lambda(r)-h L(r) .
$$

Since by Ahlfors' first covering theorem, $S\left(D_{k}\right) \leqq S(r)+h L(r)$, we have

$$
\sum_{k=1}^{i q}\left(1-\frac{1}{\mu_{k}}\right) S(r) \leqq S(r)+\lambda(r)+h L(r)
$$

so that

$$
\sum_{k=1}^{q}\left(1-\frac{1}{\mu_{k_{0}}}\right) \leqq 1+\frac{\lim }{r \rightarrow \infty} \frac{\lambda(r)}{S(r)} \leqq 2 .
$$

Hence we have the following discs-theorem :

Theorem XII. If $\left[w, w_{0}\right]<\delta$ contains a transcendental singularity of the inverse function $\varphi(w)$ of $w=f(z)$ and the Riemann surface $F$ of $\phi(w)$ ramifies $\mu_{k}$-ply above $D_{k}(k=1,2, \ldots, q)$, then

$$
\sum_{k=1}^{q}\left(1-\frac{1}{\mu_{k}}\right) \leqq 1+\lim _{r \rightarrow \infty} \frac{\lambda(r)}{S(r)} \leqq 2,
$$

where $D_{k}$ are simply. connected domains in $\left[w, w_{0}\right]<\delta$ and $r$ tends to infinity by taking the values outside certain intervals $I_{n}$, such that $\sum_{n} \int_{I_{n}} d \log r<\infty$.

Hence one of any three, four or five simply connected domains in $\left[w, w_{0}\right]<\delta$ is covered schlicht by the Riemann surface $F$, according as

$$
\lim _{r \rightarrow \infty} \frac{\lambda(r)}{S(r)}<\frac{1}{2}, \frac{1}{2} \leqq \lim _{r \rightarrow \infty} \frac{\lambda(r)}{S(r)}<1, \quad \frac{\lim }{r \rightarrow \infty} \frac{\lambda(r)}{S(r)}=1 .
$$

K. Noshiro $\left({ }^{9}\right)$ proved that $\sum_{k=1}^{q}\left(1-\frac{1}{\mu_{k}}\right) \leqq 1$, if $\Delta$ is simply connected and K. Kunugui( $\left.{ }^{10}\right)$ proved that $\sum_{k=1}^{q}\left(1-\frac{1}{\mu_{k}}\right) \leqq 2$. The general case is due to $\mathrm{Y}$. Tumura $\left({ }^{12}\right)$.

(12) Y. TumurA: 1. c. (11) also M. TsuJi : 1. c. (11). 
Remark. I. In Theorem VIII and XII, we can relace $1+\lim _{r \rightarrow \infty} \frac{\lambda(r)}{S(r)} \leqq 2$ by $1+\frac{\lim }{r \rightarrow \infty} \frac{A(r)}{T(r)} \leqq 2$, where $r$ tends to infinity by taking the values outside certain intervals $I_{n}$, such that $\sum_{n} \int_{I_{n}} d \log \log r<\infty$.

II. Similarly as Ahlfors, we can prove the defect relation :

$$
\sum_{h=1}^{q} \delta\left(D_{k}\right)+\sum_{k=1}^{q} \vartheta\left(D_{k}\right) \leqq 1+\lim _{r \rightarrow \infty} \frac{\lambda(r)}{S(r)} \leqq 2 .
$$

(E) Uniqueness theorem.

Let $\bar{n}(r, a)$ be the number of zero points of $f(z)-a$ in $A_{r}$, where the multiple zero point is counted only once and put $\bar{N}(r, a)=\int_{r_{0}}^{r} \frac{\bar{n}(r, a)}{r} d r$.

Since we have instead of (37), $(q-1) S(r) \leqq \sum_{k=1}^{q} \bar{n}\left(r, a_{k}\right)+\lambda(r)+h L(r)$, we have the second fundamental theorem in the more precise form:

$$
(q-1) T(r) \leqq \sum_{k=1}^{q} \bar{N}\left(r, a_{k}\right)+\Lambda(r)+O\left(\int_{r_{0}}^{r} \frac{L(r)}{r} d r\right)
$$

From (54), similarly as Nevanlinna ${ }^{(13)}$, we can prove the uniqueness theorem :

Theorem XIII. Let $f_{1}(z)$ and $f_{2}(z)\left(f_{1}(z) \neq f_{2}(z)\right)$ be meromorphic in $\Delta$, where $\left[f_{1}(z), w_{0}\right]<\delta,\left[f_{2}(z), w\right]<\delta$ in $\Delta$ and $\left[f_{1}(z), w_{0}\right]=\delta,\left[f_{2}(z), w_{0}\right]=\delta$ on the boundary of $A$. If $\left[w, w_{0}\right]<\delta$ contains a transcendental singularity of one of the inverse functions of $f_{1}(z), f_{2}(z)$ and $f_{1}(z)-a_{k}, f_{2}(z)-a_{k}(k=$ $1,2 \ldots, q)$ have the same zero points in $A$, then $q \leqq 3+\lim _{r \rightarrow \infty} \frac{\Lambda(r)}{T(r)} \leqq 4$. Hence if $q>3+\lim _{x_{i \rightarrow \infty}} \frac{\Lambda(r)}{T(r)}$, then $f_{1}(z) \equiv f_{2}(z)$, where $r$ tends to infinity by taking the values outside certain intervals $I_{n}$, such that $\sum_{n} \int_{I_{n}} d \log \log r<\infty$.

\section{PART II. AHLFORS' THEOREM ON THE NUMBER OF ASYMPTOTIC VALUES.}

\section{Quasi-direct transcendental singularity.}

With the same notation as in $\$ 1$, we assume that $w_{0}$ is a transcendental singularity of the inverse function $\phi(w)$ of $w=f(z)$. Then $\Delta$

(13) Nevanlinna: Le théorème de Picard-Borel et la théorie des fontions méromorphes. p. 109. 
contains a curve $C$ extending to infinity along which $\left[f(z), w_{0}\right]$ tends to zero. $w_{0}$ is called by Iversen a direct transcendental singularity of $\phi(w)$, if $f(z)-w_{0}$ has no zero point in $\Delta$. Ahlfors ${ }^{(14)}$ proved that if $f(z)$ is meromorphic for $|z|<\infty$ and of finite order $\rho$, then the number $n$ of direct transcendental singularities of $\phi(w)$ is $\leqq 2 \rho$, if $n \geqq 2$. We will show that if the number of zero points of $f(z)-w_{0}$ in $\Delta$ is not so large, then the number $n$ of such singularities is $\leqq 2 \rho$, if $n \geq 2$. For this purpose, we introduce a new notion "quasi-direct transcendental singularity" as follows.

Let $n(r)$ be the number of zero points of $f(z)-w_{0}$ in $\Delta_{r}$. We will call $w_{0}$ a quasi-direct transcendental singularity, if

$$
n(r) \leqq K \int_{r_{0}}^{r} \frac{d r}{r \bar{\theta}(r)}
$$

for any choice of $z_{0}$ and $U$, where $K$ is independent of $r$, but may depends on $z_{0}$ and $U$. Then we will prove the following theorem.

Theorem XIV. Let $f(z)$ be meromorphic for $|z|<\infty$ and of finite order $\rho$, then the number $n$ of quasi-direct transcendental singularities of the inverse function $\phi(w)$ of $w=f(z)$ is $\leqq 2 \rho$, if $n \geqq 2$.

\section{Some lemmas.}

Lemma I. If $|z|<1,|a|<1$, then

$$
\left|\frac{z-a}{1-\bar{a} z}\right| \geqq\left|\frac{|z|-|a|}{1-|a| \cdot|z|}\right| \text {. }
$$

Proof. We put $z=r e^{i \vartheta}, a=\rho e^{i \varphi}$, then

$$
\left|\frac{z-a}{1-a z}\right|^{2}-\left|\frac{|z|-|a|}{1-|a| \cdot|z|}\right|^{2}=\frac{2 r \rho\left(1-r^{2}\right)\left(1-\rho^{2}\right)(1-\cos (\theta-\varphi))}{\left(1-2 r \rho \cos (\theta-\phi)+r^{2} \rho^{2}\right)(1-r \rho)^{2}} \geqq 0,
$$

so that

$$
\left|\frac{z-a}{1-\bar{a} z}\right| \geqq\left|\frac{|z|-|a|}{1-|a| \cdot|z|}\right|
$$

Lemma II. Let $0<a_{n}<1$ and $n(r)=$ the number of $a_{n}$ such that ${ }^{n} \leqq r$ satisfy the condition:

$$
n(r) \leqq K \log \frac{1}{1-r} \quad(K=\text { const. })
$$

(14) L. Ahlfors: Über die asymptotischen Wert der meromorphen Funktionen endlicher Ordnung. Acta Acad. Aboensis. Math. et Phys. 6 (1932) or R. Nevanlinna's book p. 294. l.c. (4). 
and let

$$
F(r)=\prod_{n=1}^{\infty}\left|\frac{r-a_{n}}{1-a_{n} r}\right| \quad(0<r<1)
$$

Then there exists a sequence $\rho_{n} \rightarrow 1$, such that

$$
F\left(\rho_{n}\right) \geqq \delta>0, \quad(\delta=\text { const. }) .
$$

Proof. We put $q=e^{-\frac{1}{K}}$ and $\frac{1-a_{n}}{1-r}=\theta_{n}=\theta_{n}(r)$, then

$$
\left|\frac{r-a_{n}}{1-a_{n} r}\right|=\left|\frac{1-\theta_{n}}{1+r \theta_{n}}\right| \geqq\left|\frac{1-\theta_{n}}{1+\theta_{n}}\right|=\left|1-\frac{2 \theta_{n}}{1+\theta_{n}}\right|=\left|1-\frac{\frac{2}{\theta_{n}}}{1+\frac{1}{\theta_{n}}}\right| .
$$

If $\theta_{n} \leqq q$, then $\frac{2 \theta_{n}}{1+\theta_{n}} \leqq \frac{2 q}{1+q}<1$. Hence $\left|1-\frac{2 \theta_{n}}{1+\theta_{n}}\right| \geqq e^{-K_{1} \theta_{n}}$, if $\theta_{n} \leqq q$ and $\left|1-\frac{\frac{2}{\theta_{n}}}{1+\frac{1}{\theta_{n}}}\right| \geqq e^{-K_{1} \frac{1}{\theta_{n}}}$, if $\frac{1}{\theta_{n}} \leqq q \quad\left(K_{1}=\right.$ const. $)$, so that

$$
\begin{aligned}
\left|\frac{r-a_{n}}{1-a_{n} r}\right| & \geqq e^{-K_{1} \theta_{n}(r)}, \quad \text { if } \quad \theta_{n}(r) \leqq q, \\
& \geqq e^{-K_{1} \frac{1}{\theta_{n}(r)}}, \quad \text { if } \quad \frac{1}{\theta_{n}(r)} \leqq q .
\end{aligned}
$$

We put $r_{k}=1-q^{k}$ and let

$I_{k}: r_{k-1}<r \leqq r_{k}$ and $n_{k}=n\left(r_{k}\right)-n\left(r_{k-1}\right)=$ the number of $a_{n}$ in $I_{k}$, then since $n\left(r_{k}\right) \leqq K \log \frac{1}{q^{k}}=K k \log \frac{1}{q}=k$, we have

$$
n_{1}+n_{2}+\cdots+n_{k}=n\left(r_{k}\right) \leqq k, \quad n_{k} \leqq k .
$$

Let $N \geqq 5$ and we divide $I_{k}$ into two classes $I_{k}^{\prime}$ and $I_{k}^{\prime \prime}$, such that we denote $I_{k}$ by $I_{k}{ }^{\prime}$, if $n_{k} \geqq N$ and by $I_{k}{ }^{\prime \prime}$, if $n_{k}<N$.

We take $\left[\frac{n_{k}}{N}\right]$ consecutive intervals $I_{s}$ on the left and on the right of $I_{k}^{\prime}$ and denote this intervals-group by $I_{(k)}$ :

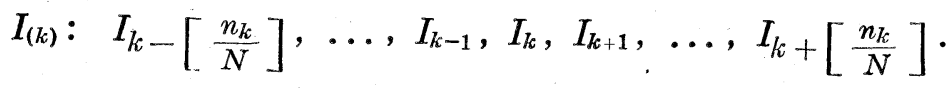


Then $s_{k}=$ the number of intervals in $I_{(k)}=2\left[\frac{n_{k}}{N}\right]+1 \leqq 3 \frac{n_{k}}{N}$.

Since

$$
\frac{3}{N}\left(n_{1}+n_{2}+\cdots+n_{k}\right) \leqq \frac{3 k}{N}
$$

we see that

at most $\frac{3 k}{N}$ intervals among $I_{1}, \ldots, I_{k}$ are coverd by the intervals-groups $I_{(\nu)}(\nu \leqq k)$.

Next we consider $\left.I_{(k+\nu}\right)(\nu \geq 1)$. Its left end interval is $I_{k+\nu}-\left[\frac{n_{k+\nu}}{N}\right]$.

Now

$$
\begin{aligned}
k+\nu-\left[\frac{n_{k+\nu}}{N}\right] \geqq k+\nu-\frac{n_{k+\nu}}{N} \geqq k+\nu-\frac{k+\nu}{N} & =(k+\nu)\left(1-\frac{1}{N}\right) \\
& \geqq k\left(1-\frac{1}{N}\right) .
\end{aligned}
$$

Hence

at most $\frac{k}{N}$ intervals among $I_{1}, \ldots, I_{k}$ are covered by $I_{(k+\nu)}$

$$
(\nu \geqq 1) \text {. }
$$

Consequently from (59) and (60), we see that at least $\left(1-\frac{4}{N}\right) k$ intervals among $I_{1}, \ldots, I_{k}$ are uncovered by $I_{(\nu)}(\nu=1,2, \ldots)$.

Let $I_{i}$ be an uncovered interval. There are $s=j-i-1$ intervals between $I_{i}$ and $I_{j}^{\prime}(j \geqq i+2)$, so that $s \geqq\left[\frac{n_{j}}{N}\right] \geqq \frac{n_{j}}{N}-1$, hence $n_{j} \leqq N(s+1)$.

If $r \in I_{i}, \quad a_{n} \in I_{j}^{\prime}$, then $\theta_{n}(r)=\frac{1-a_{n}}{1-r} \leqq q^{s}$, so that for $r \in I_{i}$,

$$
\sum_{a_{n} \in I_{j}^{\prime}} \theta_{n}(r) \leqq n_{j} q^{s} \leqq N(s+1) q^{s}
$$

so that 


$$
\sum_{j=i+2}^{\infty} \sum_{a_{n} \in I_{j}^{\prime}} \theta_{n}(r) \leqq N \sum_{s=0}^{\infty}(s+1) q^{s}=A . N \text { (say). }
$$

Since $n_{j}<N$ in $I_{j}^{\prime \prime}(j \geqq i+2)$, we have for $r \varepsilon I_{i}$,

$$
\sum_{a_{n} \in I_{j}^{\prime \prime}} \theta_{n}(r) \leqq n_{j} q^{s} \leqq N q^{s},
$$

so that

$$
\sum_{j=i+2}^{\infty} \sum_{a_{n} \in I_{j}^{\prime \prime}} \theta_{n}(r) \leqq N \sum_{s=0}^{\infty} q^{s}<A . N
$$

Hence from (61), (62),

$$
\sum_{j=i+2}^{\infty} \sum_{a_{n} \in I_{j}} \theta_{n}(r) \leqq 2 A . N
$$

Similarly

$$
\sum_{j=1}^{i-2} \sum_{a_{n} \in I_{j}} \frac{1}{\theta_{n}(r)} \leqq 2 A . N
$$

We put $I_{i}^{0}=I_{i-1}+I_{i}+I_{i+1}$, then from (55), (56), (63), (64), we have for $r \in I_{i}$,

$$
\prod_{a_{n} \bar{\epsilon} I_{i}^{0}}\left|\frac{r-a_{n}}{1-a_{n} r}\right| \geqq e^{-4 A K_{1} N},
$$

where $a_{n} \bar{\epsilon} I_{i}^{0}$ means that $a_{n}$ does not belong to $I_{i}^{0}$.

Since $I_{i}^{0}$ contains at most $3 N$ of $a_{n}$ 's, we can find $\rho_{i}$ in $I_{i}$, such that

$$
\prod_{a_{n} \in I_{i}^{0}}\left|\frac{\rho_{i}-a_{n}}{1-a_{n} \rho_{i}}\right| \geqq K_{0}=K_{0}(N) .
$$

From (65), (66), we have

$$
F\left(\rho_{i}\right) \geqq K_{0} e^{-\alpha N} \quad\left(\alpha=4 A K_{1}\right) .
$$

Since the number of uncovered intervals increases indefinitely with $k$, we have a sequence $\rho_{i} \rightarrow 1$ for which (67) holds, which proves our lemma.

Remark. If we apply H. Cartan's theorem on $\prod_{a_{n} \in I_{i}^{0}}\left|\frac{r-a_{n}}{1-a_{n} r}\right|$, we can prove that $F(\rho) \geqq e^{-\alpha N \log N}$ holds in $0 \leqq \rho \leqq r$, except certain 
intervals $I_{n}$, such that $\sum_{n} \int_{I_{n}} d \log \frac{1}{1-\rho} \leqq \frac{1}{N} \log \frac{1}{1-r}$, where $\alpha$ is a constant independent of $N$.

Theorem XV. Let $0<\left|a_{n}\right|<1$ and $n(r)=$ number of $a_{n}$ in $|z| \leqq r$ satisfy the condition:

$$
n(r) \leqq K \log \frac{1}{1-r} \quad(K=\text { const. })
$$

and let

$$
F(z)=\prod_{n=1}^{\infty} \frac{\bar{a}_{n}}{\left|a_{n}\right|} \cdot \frac{a_{n}-z}{1-\bar{a}_{n} z} \quad(|z|<1) .
$$

Then there exists a sequence $\rho_{n} \rightarrow 1$, such that

$$
\underset{|z|=\rho_{n}}{\operatorname{Min}}|F(z)| \geqq \delta>0 \quad(\delta=\text { const. }) .
$$

Proof. By Lemma I, $|F(z)| \geq \prod_{n=1}^{\infty}\left|\frac{|z|-\left|a_{n}\right|}{1-\left|a_{n}\right| \cdot|z|}\right|$, so that the theorem follows from Lemma II.

\section{Proof of Theorem XIV.}

We assume that $w_{0}=\infty$ is a quasi-direct transcendental singularity of $\varphi(w)$ and a $\delta$-neighbourhood $U$ of $w_{0}$ correspond to $\Delta$ on the $z$-plane, so that $|f(z)|>\lambda$ in $\Delta$ and $|f(z)|=\lambda$ on the boundary $A$ of $\Delta$. We suppose that there exists a boundary curve of $\Delta$, which extends to infinity. Let $z_{0}$ be a point on the outermost boundary of $\Delta$ and we take $r_{0}$ so small that $\left|z-z_{0}\right|=r_{0}$ meets $A$ at two points $z_{1}, z_{2}$, so that the common part of $\left|z-z_{0}\right| \leqq r_{0}$ and $\Delta$ is a simply connected domain and let $\theta_{r_{0}}$ be the common part of $\left|z-z_{0}\right|=r_{0}$ and $\Delta$ and $\bar{A}$ be the boundary of $\bar{A}$. We map $\bar{A}$ on $|\zeta|<1$, such that $z_{0}, z, z_{2}$ become $\zeta=-1, i,-i$. We denote the image of $\theta_{r_{0}}$ in $|\zeta|<1$ by $\sigma_{r_{0}}$.

Let $z^{\prime}$ be point on $\bar{A}$, such that $\left|z^{\prime}-z_{0}\right|=r^{\prime} \geqq 2 r_{0}$ and let $z^{\prime}$ correspond to $\zeta^{\prime}=e^{i \varphi}\left(|\phi|<\frac{\pi}{2}\right)$ on $|\zeta|=1$. Next we map $|\zeta|<1$ on $|x|<1$, such that $\zeta=-1, i, \zeta^{\prime}$ become $x=-1, i, 1$, so that $\frac{1+x}{1-x}=i \frac{\zeta+1}{\zeta-\zeta^{\prime}} \cdot \frac{i-\zeta^{\prime}}{i+1}$. Hence

$$
\left|\frac{1+x}{1-x}\right| \leqq \frac{4}{\left|\zeta-\zeta^{\prime}\right|}
$$

If we denote the image of $\sigma_{r_{0}}$ in $|x|<1$ by $\gamma_{r_{0}}$, then on $\gamma_{r_{0}}$, 


$$
\left|\frac{1+x}{1-x}\right| \geqq \eta>0
$$

where $\eta$ is a constant independent of $z^{\prime}$. Next by $w=u+i v=\log \frac{1+x}{1-x}$, we map $|x|<1$ on an infinite strip $S:-\infty<u<\infty,|v|<\frac{\pi}{2}$, such that $x=-1,1$ correspond to $w=-\infty, \infty$ and let $\gamma_{r_{0}}$ correspond to $\lambda r_{0}$ in $S$.

Hence by (69), we have on $\lambda_{r_{0}}$,

$$
\Re(w)=\log \left|\frac{1+x}{1-x}\right| \geqq \log \eta
$$

We connect $z_{0}$ and $z^{\prime}$ by a curve $L$ in $\Delta$ and let $\left|z-z_{0}\right|=r\left(r^{\prime}>r>r_{0}\right)$ intercept $\bar{\Delta}$ in a finite number of cross cuts, at least one of which separates $z_{0}$ and $z^{\prime}$ in $\bar{s}$. Let $C(r)$ be such a cross cut, which meets $L$ at first, when we proceed from $z_{0}$ to $z^{\prime}$ on $L$. If we denote the length of $C(r)$ by $r \bar{\theta}_{1}(r)$, then $r \bar{\theta}_{1}(r) \leqq r \bar{\theta}(r)$. Now by Ahlfors' Verzerrungssatz we have on any point of the image of $C(r)$ in $S$,

$$
\Re(w)=\log \left|\frac{1+x}{1-x}\right|>\pi \int_{r_{0}}^{r} \frac{d r}{r \bar{\theta}(r)}+\log \eta-4 \pi, \text { if } \int_{r_{0}}^{r} \frac{d r}{r \bar{\theta}(r)}>2 .
$$

Hence by (68), we have on the image of $C(r)$ in $|\zeta|<1$,

$$
\left|\zeta-\zeta^{\prime}\right| \leqq K_{0} e^{-\pi \int_{r_{0}}^{r} \frac{d r}{r \bar{\theta}(r)}} \quad\left(K_{0}=\text { const. }\right)
$$

Let $a_{n}$ be the poles of $f(z)$ in $\Delta$, then since $w_{0}=\infty$ is a quasi-direct transcendental singularity of $\phi(w)$,

$$
n(r) \leqq K \int_{r_{0}}^{r} \frac{d r}{r \bar{\theta}(r)} \quad(K=\text { const. })
$$

where $n(r)$ is the number of $a_{n}$ in $\Delta_{r}$.

We put $\left|a_{\nu}-z_{0}\right|=r_{\nu}$ and suppose that $r_{m}=r_{m+1}=\cdots=r_{n}$. Let $C_{1}, \ldots, C_{k}$ be the cross cuts of $\overline{4}$, which lie on $\left|z-z_{0}\right|=r_{n}$ and let $a_{\nu}$, $C_{i}$ correspond to $\zeta_{\nu}, \Gamma_{i}$ in $|\zeta|<1$.

Now $\Gamma_{i}$ separates $|\zeta|<1$ into two domains $D_{i}, D_{i}^{\prime}$, where $D_{i}^{\prime}$ contains -1 on its boundary. $D_{i}, D_{j}(i \neq j)$ may have no common points or $D_{i}$ is contained in $D_{j}$. We assume that $D_{1}$ is not contained in any $D_{i}$ and contains $D_{2}, \ldots, D_{s}$. Then we see that there exists a point $\zeta^{\prime}$ on $|\zeta|=1$, which lies on the boundary of $D_{1}$ and lies outside $D_{2}, \ldots, D_{c}$. 
Let $\zeta^{\prime}$ correspond to $z^{\prime}$ on $\bar{\Lambda}$, then $\left|z^{\prime}-z_{0}\right|>r_{n}$. Hence by putting $r=r_{n}$ in (71), (72), we, have on $\Gamma_{1}$ and hence in $D_{1}$,

$$
\left|\zeta-\zeta^{\prime}\right| \leqq e^{-K_{1} n} \quad\left(K_{1}=\text { const. }\right) .
$$

Hence if $\zeta_{\nu}(m \leqq \nu \leqq n)$ is contained in $D_{1}$,

$$
1-\left|\zeta_{\nu}\right| \leqq\left|\zeta_{\nu}-\zeta^{\prime}\right| \leqq e^{-K_{1} n} \leqq e^{-K_{1} \nu},
$$

so that for any $n\left(n \geq n_{0}\right)$

$$
1-\left|\zeta_{n}\right| \leqq e^{-K_{1} n}
$$

From (74), we see easily that

$$
n_{1}(r) \leqq A \log \frac{1}{1-r} \quad(A=\text { const. })
$$

where $n_{1}(r)$ is the number of $\zeta_{n}$ in $|\zeta| \leqq r$. Hence by Theorem XV, there exists a sequence $\rho_{n} \rightarrow 1$, such that

where

$$
\operatorname{Min}_{|\zeta|=\rho_{n}}|g(\zeta)| \geqq \delta>0 \quad(\delta=\text { const. }) \text {, }
$$

$$
g(\zeta)=\prod_{n=1}^{\infty} \frac{\bar{\zeta}_{n}}{\left|\zeta_{n}\right|} \cdot \frac{\zeta_{n}-\zeta}{1-\bar{\zeta}_{n} \zeta}
$$

If we put $G(z)=g(\zeta)$, then $|G(z)|<1$ in $\Delta$ and $G\left(a_{n}\right)=0$, so that $F(z)=f(z) G(z)$ is regular in $\Delta$ and $|F(z)| \leqq|f(z)|$ in and on the boundary of $\Delta . F(z)$ is unbounded in $\Delta$. For, if $F(z)$ is bounded and $|F(z)| \leqq K$ in $\Delta$, then $|G(z)| \leqq \frac{K}{|f(z)|}$. By the hypothesis, $f(z) \rightarrow \infty$ along $C$, so that $G(z) \rightarrow 0$ along $C$, which contradicts (76). Hence $F(z)$ is unbounded in $A$. Next we map $\bar{\Delta}$ on the half-plane $\Im(\dot{\zeta})>0$ and put $\log \frac{|w(z)|}{\lambda}=u(\zeta)$, where $w(z)=F(z)=f(z) G(z)$.

Since $u(\zeta)$ is unbounded in $\Im(\zeta)>0$, there exists a point $\zeta_{0}$ in $\Im(\zeta)>0$, such that $u\left(\zeta_{0}\right)>0$. This point established, we proceed' similarly as Ahlfors' proof and we obtain

$$
\pi \int_{r_{0}}^{r} \frac{d r}{r \overline{\bar{\theta}}(r)}<\log \log M(r)+\text { const. }
$$

where $M(r)=\operatorname{Max}_{\theta_{r}}|w(z)|$. 
Let $U(z)$ be a harmonic function in $\left|z-z_{0}\right|<r^{\prime}\left(r^{\prime}>r\right)$, such that $U(z)=\log |f(z)|$ on $\theta_{r^{\prime}}$ and $U(z)=\log \lambda$ on the remaining part of $\left|z-z_{0}\right|=r^{\prime}$. Since $\log |w(z)| \leqq \log \lambda \leqq U(z)$ on the boundary of $\Delta_{r^{\prime}}$, we have $\log |w(z)| \leqq U(z)$ in $A_{r^{\prime}}$. Hence, if $M(r)=\left|w\left(z_{0}+r e^{i \varphi}\right)\right|$, we have

$$
\begin{aligned}
& \log M(r) \leqq \frac{1}{2 \pi} \int_{0}^{2 \pi} U\left(z_{0}+r^{\prime} e^{i \vartheta}\right) \frac{r^{\prime 2}-r^{2}}{r^{\prime 2}-2 r r^{\prime} \cos (\theta-\varphi)+r^{2}} d \theta \\
& \quad \leqq \frac{r^{\prime}+r}{r^{\prime}-r} \int_{\theta_{r^{\prime}}} \log \left|f\left(z_{0}+r^{\prime} e^{i \theta}\right)\right| d \theta+\text { const. } \leqq \frac{r^{\prime}+r}{r^{\prime}-r} m\left(r^{\prime}, \infty\right)+\text { const. }
\end{aligned}
$$

Hence for $r^{\prime}=2 r, \log M(r) \leqq 3 T(2 r)+$ const., so that

$$
\pi \int_{r_{0}}^{r} \frac{d r}{r \overline{\bar{\theta}}(r)}<\log T(2 r)+\text { const. }
$$

From this we have $n \leqq 2 \rho$ by the well known reasonings.

Remark. If $T(r)=O\left((\log r)^{2}\right)$, then the order of $f(z)$ is zero and let $n\left(r, w_{0}\right)$ be the number of zero points of $f(z)-w_{0}$ in $|z| \leqq r$. Then since $n\left(r, w_{0} \log r \leqq \int_{r}^{r^{2}} \frac{n\left(r, w_{0}\right)}{r} d r \leqq N\left(r^{2}, w_{0}\right) \leqq T\left(r^{2}\right)=O\left((\log r)^{2}\right), \quad n\left(r, w_{0}\right)\right.$ $=O(\log r)$. Since $\log r-\log r_{0} \leqq 2 \pi \int_{r_{0}}^{r} \frac{d r}{r \bar{\theta}(r)}$, it follows that every transcendental singularity of $\varphi(w)$ is quasi-direct, hence we have the following Valiron's theorem. $\left({ }^{15}\right)$

Theorem XVI. If $T(r)=O\left((\log r)^{2}\right)$, then there exists at most one asymptotic value.

Y. Tumura $\left({ }^{16}\right)$ proved that $T(r)=O\left((\log r)^{2}\right)$ can be replaced by $\lim _{r \rightarrow \infty} \frac{T(r)}{(\log r)^{2}}<\infty$.

\section{Extension of Theorem XIV.}

Let $f(z)$ satisfy the condition in $\S 1$ and be of finite order $\rho$ in $\Delta$. We will investigate the number of quasi-direct transcendental singularities of the inverse function of $f(z)$ in $\left[w, w_{0}\right]<\delta$.

(15) G. VALiRon: Súr les valeurs asymptotiques de quelque fonction méromorphes. Rःndiconti mat. Palermo. 46 (1925). Sur le nombre des singularités transcendantes des fonctions inverse d'une classe d'algebroide. C.R. 200 (1935).

(16) Y. Tumura: Sur les théorèmes de M. Valiron et les singularités transcendantes indirectement critiques. Proc. Imp. Acad. 17 (1941). 
Theorem XVII. Let $f(z)$ be meromorphic and of finite order $\rho$ in $\Delta$, where $\left[f(z), w_{0}\right]<\delta$ in $\Delta$ and $\left[f(z), w_{0}\right]=\delta$ on the boundary $A$ of $\Delta$ and $n$ be the number of quasi-direct transcendental singularities of the inverse function $\phi(w)$ of $w=f(z)$ in $\left[w, w_{0}\right]<\delta$. Then $n \leqq 2 \rho$, when $A$ contains $a$ curve extending to infinity and $n \leqq 2 \rho$, if $n \geqq 2$, when $A$ consists of only closed curves.

Proof. Let $\left[w, w_{0}\right]<\delta$ contain $n$ quasi-direct transcendental singularities, $a_{1}, \ldots, a_{n}$ of $\phi(w)$ and $a \delta_{i}$-neighbourhood $U_{i}$ of $\alpha_{i}$ correspond to $\Delta_{i}$ on the $z$-plane. $\left({ }^{17}\right)$ We define $\overline{\overline{\Delta_{i}}}, r \overline{\overline{\theta_{i}}}(r), T\left(r, a_{i}, w ; \Delta_{i}\right)$ etc as in $\S 1$. We take $\delta_{i}$ so small that $\overline{\overline{\bar{J}_{i}}}$ do not overlap, so that $\overline{\bar{\theta}}_{1}(r)+\cdot+\overline{\bar{\theta}}_{n}(r) \leqq 2 \pi$.

By (78), we have

$$
\pi \int_{r_{0}}^{r} \frac{d r}{r \bar{\theta}_{i}(r)}<\log T\left(2 r, a_{i}, w ; \Delta_{i}\right)+\text { const. }
$$

From $\left(1^{*}\right)$,

$$
\begin{aligned}
T\left(2 r, a_{i}, w ; \Delta_{i}\right) & \leqq T\left(2 r, a_{i}, w ; \Delta\right) \\
& =T(2 r, w ; \Delta)+O\left(r^{\rho^{\prime}}\right)<r^{p+\varepsilon},
\end{aligned}
$$

so that

$$
\pi \int_{r_{0}}^{r} \frac{d r}{r \overline{\bar{\theta}}_{i}(r)}<(\rho+\varepsilon) \log r+\text { const. }
$$

Since $\frac{1}{\overline{\bar{\theta}}_{1}(r)}+\cdot+\frac{1}{\overline{\bar{\theta}}_{n}(r)} \geq \frac{n^{2}}{\overline{\bar{\theta}}_{1}(r)+\cdot+\overline{\bar{\theta}}_{n}(r)} \geq \frac{n^{2}}{2 \pi}$, we have $n \log r \leqq 2(\rho+\varepsilon) \log r+$ const. Hence $n \leqq 2 \rho$.

Theorem XVIII. Let $f(z)$ be meromorphic and of finite order $\rho$ in $\Delta$, where $\left[f(z), w_{0}\right]<\delta$ in $\Delta$ and $\left[f(z), w_{0}\right]=\delta$ on the boundary of $\Delta$ and $n$ be the number of quasi-direct transcendental singularities of the inverse function $\phi(w)$ of $w=f(z)$ in $\left[w, w_{0}\right]<\delta$. We assume that the boundary of 4 contains a curve extending to infinity. Then

$$
n \leqq \frac{\rho}{\pi} / \varlimsup_{r \rightarrow \infty} \frac{1}{\log r} \int_{r_{0}}^{r} \frac{d r}{r \bar{\theta}(r)} \leqq \frac{\bar{\theta} \rho}{\pi} \text {, where } \bar{\theta}=\varlimsup_{r \rightarrow \infty} \bar{\theta}(r)
$$

Proof. Suppose that $f(z)$ is regular in $\Delta$, where $|f(z)|>\lambda$ in $\Delta$ and $|f(z)|=\lambda$ on the boundary of $\Delta$ and $\Delta$ contains only one asymptotic path for the value $\infty$, so that $f(z)$ tends to infinity along a curve $C$, which

(17) $\Delta_{i}$ has boundary curves :extending to infinity, if $A$ has an infinite branch or when $\Lambda$ consists of only closed curves, if $n \geqq 2$. 
connects a point $z_{0}$ on the boundary $\Lambda$ of $\Delta$ with $z=\infty$ in $\Delta$. We map $\bar{\Delta}$ on $\Im(\zeta)>0$ by $\zeta=g(z)(\infty=g(\infty))$ and let the image of $\Delta$ and $C$ in $\Im(\zeta)>0$ be denoted by $D$ and $\gamma$ respectively and put $F(\zeta)=f(z)$. Then $F(\zeta) \rightarrow \infty$, when $\zeta$ tends to $\infty$ along $\gamma$. Now by the mapping $\zeta=g(z), z=\infty$ corresponds to a closed set $E$ of measure zero on the real axis $\Im(\zeta)=0$. At any point $(\neq \infty)$ on $\mathfrak{J}(\zeta)=0, F(\zeta)$ is bounded, since atherwise, there would exist another asymptotic path for the value $\infty$, contradictory to the hypothesis. Let $\zeta_{1}(\neq \infty)$ be a point on $E$ and its neighbourhood in $D$ be denoted by $U$. Then considering on the $z$-plane we can easily prove that $\lim _{\zeta \rightarrow \zeta_{1}}|F(\zeta)| \leqq \lambda$, when $\zeta$ tends to $\zeta_{1}$ in $U$. Hence, since $|F(\zeta)|>\lambda$ in $U$, we have

$$
\lim |F(\zeta)|=\lambda,
$$

when $\zeta$ tends to any point $(\neq \infty)$ on the real axis.

From (80), similarly as we have deduced (78), we have

$$
\pi \int_{r_{0}}^{r} \frac{d r}{r \bar{\theta}(r)}<\log T(2 r, \infty, w ; \Delta)+\text { const. }
$$

Similarly as in $\$ 9$ we can prove that (81) holds if the number of poles of $f(z)$ in $\Delta_{r}$ is $\leqq K \int_{r_{0}}^{r} \frac{d r}{r \bar{\theta}(r)}$.

Now by Theorem XVII, the number of quasi-direct transcendental singularities of $\phi(w)$ is finite. Let $a_{1}, \ldots, a_{n}$ be such singularities in $\left[w, w_{0}\right]<\delta$ and let a $\delta_{i}$-neighbourhood $U_{i}$ of $a_{i}$ correspond to $\Delta_{i}$ on the $z$-plane, where we take $\delta_{\boldsymbol{i}}$ so small that $\overline{\bar{A}_{i}}$ do not overlap. Hence $\bar{\theta}_{1}(r)+\cdot+\bar{\theta}_{n}(r) \leqq \bar{\theta}(r)$ and there is only one asymptotic path for the value $a_{i}$ in $\Delta_{i}$. Then from (81),

$$
\pi \int_{r_{0}}^{r} \frac{d r}{r \bar{\theta}_{i}(r)}<\log T\left(2 r, a_{i}, w ; \Delta_{i}\right)+\text { const. }
$$

Similarly as (79), we have from (82),

$$
\pi \int_{r_{0}}^{r}\left(\frac{1}{\bar{\theta}_{1}(r)}+\cdot+\frac{1}{\bar{\theta}_{n}(r)}\right) \frac{d r}{r} \leqq n(\rho+\varepsilon) \log r+\text { const., }
$$

Since

$$
\begin{gathered}
\frac{1}{\bar{\theta}_{1}(r)}+\cdot+\frac{1}{\bar{\theta}_{n}(r)} \geqq \frac{n^{2}}{\bar{\theta}_{1}(r)++\bar{\theta}_{n}(r)} \geqq \frac{n^{2}}{\bar{\theta}(r)}, \\
\pi n \int_{r_{0}}^{r} \frac{d r}{r \bar{\theta}(r)} \leqq(\rho+\varepsilon) \log r+\text { const. . }
\end{gathered}
$$


Hence

where

$$
n \leqq \frac{\rho}{\pi} / \varlimsup_{r \rightarrow \infty} \frac{1}{\log r} \int_{r_{0}}^{r} \frac{d r}{r \bar{\theta}(r)} \leqq \frac{\bar{\theta} \rho}{\pi},
$$

$$
\bar{\theta}=\lim _{r \rightarrow \infty} \bar{\theta}(r), \quad \text { q. e. d. }
$$

\section{Number of asymptotic values of a regular function of finite order in $\Delta$.}

Theorem XIX. Let $f(z)$ be regular and of finite order $\rho$ in $J$, where $|f(z)|>\lambda$ in $\Delta$ and $|f(z)|=\lambda$ on the boundary of $\Delta$.

Let $n$ and $n^{\prime}$ be the number of asymptotic pathes for the values a $(\lambda<|a|<\infty)$ and the value $\infty$ respectively. Then

(A) when the boundary of 4 contains a curve extending to infinity,

(i) $n^{\prime} \leqq \frac{\rho}{\pi} / \varlimsup_{r \rightarrow \infty} \frac{1}{\log r} \int_{r_{0}}^{r} \frac{d r}{r \bar{\theta}(r)} \leqq \frac{\bar{\theta} \rho}{\pi}$, where $\bar{\theta}=\varlimsup_{r \rightarrow \infty} \bar{\theta}(r)$,

(ii) $n \leqq n^{\prime}-1$.

(B) when the boundary of 1 consists of only closed curves,

(i) $n^{\prime} \leqq 2 \rho$, if $n \geqq 2$,

(ii) $n \leqq n^{\prime}$.

Proof. By the hypothesis, $w=\infty$ is a direct transcendental singulality of $\phi(w)$, so that (i) follows from Theorem XVIII. It remains to prove (ii). We will first prove (A) (ii).

We map $\bar{J}$ on $|\zeta|<1$ by $z=g(\zeta)$ and let $F(\zeta)=f(z)$. Then $F(\zeta)$ tends to infinity along curves $\gamma_{1}, \ldots, \gamma_{n^{\prime}}$ in $|\zeta|<1$ ending at $\zeta_{1}, \ldots, \zeta_{n^{\prime}}$ on $|\zeta|=1$, where some of $\zeta_{i}$ may coincide.

(i) Let $a(\lambda<|a|<\infty)$ be an asymptotic value of $F(\zeta)$, then $F(\zeta)$ tends to $a$ along a curve $\gamma$ ending at a point $\zeta_{0}$ on $|\zeta|=1$. By (80), $\zeta_{0}$ must coincide with one of $\zeta_{i}$. Let $\zeta_{0}=\zeta_{1}$ and $\Omega$ be the neighbourhood of $\zeta_{1}$ in $D$, which is the image of $\Delta$ in $|\zeta|<1$ by $z=g(\zeta)$. Now $\gamma$ divides $\Omega$ into two parts $\Omega_{1}$ and $\Omega_{2}$. We will show that $F(\zeta)$ is unbounded in each $\Omega_{i}$. For, suppose that $F(\zeta)$ is bounded in $\Omega_{1}$. Then since $\zeta_{1}$ is a regular point for the Dirichlet problem for $\Omega_{1}$, if $\varlimsup i m|F(\zeta)| \leqq m$, when $\zeta$ tends to $\zeta_{1}$ on the boundary of $\Omega_{1}$, then $\varlimsup|F(\zeta)| \leqq m$, when $\zeta$ tends to $\zeta_{1}$ in $\Omega_{1}$. Hence if we denote the cluster set of $F(\zeta)$ by $E_{1}$ and $E$, when $\zeta$ tends to $\zeta_{1}$ on the boundary of $\Omega_{1}$ and in $\Omega_{1}$ respectively, then by Hössjer's theorem, ${ }^{(18)} E$ is contained in $E_{1}$. Now

(18) G. HössJer: Bemerkung über einen Satz von E. LindelöF. Kungl. Fysiografiska Säälskapets I Lund. Förhandligar 6 (1935). 
$E_{1}$ consists of $a$ and $|w|=1$, so that $E$ consists of $a$ and $|w|=1$. But we see easily that $E$ contains a continuum connecting $a$ and $|w|=1$, which is a contradiction. Hence $F(\zeta)$ is unbounded in $\Omega_{1}$, so that $\Omega_{1}$ contains a curve along which $F(\zeta)$ tends to infinity. Similarity for $\Omega_{2}$.

(ii) Next suppose that there are two asymptotic pathes $\gamma, \gamma^{\prime}$ for the values $a$ and $b(\lambda<|a|<\infty, \lambda<|b|<\infty, a \neq b)$ ending at $\zeta_{1}$, so that $F(\zeta) \rightarrow a$ along $\gamma$ and $F(\zeta) \rightarrow b$ along $\gamma^{\prime}$. Then $F(\zeta)$ is unbounded in the neighbourhood $\Omega$ of $\zeta_{1}$ in $D$ between $\gamma$ and $\gamma^{\prime}$. For, suppose that $F(\zeta)$ is bounded in $\Omega$, then as before by Hössjer's theorem, $E_{1}$ and hence $E$ consists of $a$ and $b$ and $|w|=1$ (when infinitely many holes cluster at $\left.\zeta_{1}\right)$. But we see easily that $E$ contains a continuum connecting $a$ and $b$, which is a contradiction. Hence $F(\zeta)$ is unbounded in $\Omega$, so that $\Omega$ contains a curve along which $F(\zeta)$ tends to infinity.

From (i) and (ii) we conclude that $n \leqq n^{\prime}-1$, q. e. d.

Similarly we can prove (B) (ii).

Remark. We assumed that $f(z)$ is regular in $\lrcorner$, but if the number of poles of $f(z)$ in $\Delta$ is finite, then the same result holds, since, then $w=\infty$ is a quasi-direct transcendental singularity of $\varphi(w)$, so that $n^{\prime}$ is finite and $f(z)$ is regular in the neighbourhood of the boundary of $\Delta$. From Theorem XIX we deduce the following theorem.

Theorem XX. Let $f(z)$ be meromorphic and of finite order $\rho$ in $\Delta$, where $\left[f(z), w_{0}\right]<\delta$ in $\Delta$ and $\left[f(z), w_{0}\right]=\delta$ on the boundary of $\Delta . W e$ assume that the boundary of 4 contains a curve extending to infinity. Then $f(z)$ takes any value in $\left[w, w_{0}\right]<\delta$, except at most one value, infinitely many times in $\Delta$.

Proof. We may assume that $\left[w, w_{0}\right]<\delta$ is of the form $|w|>\lambda$, since by the rotation of the Riemann shpere, we can bring $\left[w, w_{0}\right]<\delta$ into the from $|w|>\lambda$ and the order of $f(z)$ is invariant by the rotation. Suppose that $f(z)-a$ and $f(z)-b(\lambda<|a|, \lambda<|b|, a \neq b)$ have a finite number of zero points in $\Delta$, then

$$
F(z)=U(f(z))=\frac{\bar{a} f(z)-\lambda^{2}}{f(z)-a}, \quad F_{1}(z)=\dot{U}_{1}(f(z))=\frac{\bar{b} f(z)-\lambda^{2}}{f(z)-b}
$$

have a finite number of poles in $A$ and $|F(z)|>\lambda,\left|F_{1}(z)\right|>\lambda$ in $\Delta$ and $|F(z)|=\lambda,\left|F_{1}(z)\right|=\lambda$ on the boundary of 4 . Since $U(w)$ and $U_{1}(w)$ make $|w|>\lambda$ invariant, $F(z)$ and $F_{1}(z)$ are of the same order as $f(z)$ by Theorem VIII.

Let $n$ and $n^{\prime}$ be the number of asymptotic pathes for the values $a$ $(\lambda<|a|<\infty)$ and the value $\infty$ of $F(z)$ respectively and $n_{1}$ and $n_{1}^{\prime}$ be the corresponding numbers for $F_{1}(z)$. Then by Theorem XIX, 


$$
\begin{aligned}
& n \leqq n^{\prime}-1, \\
& n_{1} \leqq n_{1}^{\prime}-1 .
\end{aligned}
$$

As we see easily,

so that

$$
n^{\prime} \leqq n_{1}, \quad n_{1}^{\prime} \leqq n
$$

$$
n^{\prime} \leqq n_{1} \leqq n_{1}^{\prime}-1 \leqq n-1 \leqq n^{\prime}-2 \text { or } \quad 0 \leqq-2,
$$

which is absurd. Hence $f(z)$ takes any value in $|w|>\lambda$, except at most one value, infinitely many times in $\Delta$.

We know from Theorem VIII, that $f(z)$ takes any value in $\left[w, w_{0}\right]<\delta$, except at most two values, infinitely many times in $\lrcorner$. We see from the above theorem that the exceptional case may occur only when the niveau curve $\left[f(z), w_{0}\right]=\delta$ consists of infinitely many closed curves clustering to infinity.

From Theorem IV, we have the following special case.

Theorem XXI. Let $f(z)$ be regular in $\Delta$, where $|f(z)|>\lambda$ in $\Delta$ and $|f(z)|=\lambda$ on the boundary of $\Delta$. We assume that the boundary of $\mathcal{A}$ contains a curve extending to infinity. If $\varlimsup_{r \rightarrow \infty} \frac{\log \log M(r)}{\log r}<\infty$, where $M(r)=\operatorname{Max}_{\theta_{r}}|f(z)|$, then $f(z)$ takes any value $a(\lambda<|a|<\infty)$ infinitely many times in $\Delta$.

Mathematical Institute, Tokyo Imperial University. 